REPRESENTATION THEORY

An Electronic Journal of the American Mathematical Society

Volume 13, Pages 309-347 (July 28, 2009)

S 1088-4165(09)00346-X

\title{
A DIAGRAMMATIC APPROACH TO CATEGORIFICATION OF QUANTUM GROUPS I
}

\author{
MIKHAIL KHOVANOV AND AARON D. LAUDA
}

\begin{abstract}
To each graph without loops and multiple edges we assign a family of rings. Categories of projective modules over these rings categorify $U_{q}^{-}(\mathfrak{g})$, where $\mathfrak{g}$ is the Kac-Moody Lie algebra associated with the graph.
\end{abstract}

\section{INTRODUCTION}

The goal of this paper is to categorify $U^{-}=U_{q}^{-}(\mathfrak{g})$, for an arbitrary simplylaced Kac-Moody algebra $\mathfrak{g}$. Here $U^{-}$stands for the quantum deformation of the universal enveloping algebra of the "lower-triangular" subalgebra of $\mathfrak{g}$.

Following the discovery of quantum groups $U_{q}(\mathfrak{g})$ by Drinfeld [12] and Jimbo [18, Ringel [41] found a Hall algebra interpretation of the negative half $U^{-}$of the quantum group in the simply-laced Dynkin case. Lusztig [31, 32, 33] gave a geometric interpretation of $U^{-}$and produced a canonical basis there via a sophisticated approach which required the full strength of the theory of $l$-adic perverse sheaves. Kashiwara [19] defined a crystal basis of $U^{-}$at 0 , a graph equipped with extra data, and in 20] constructed the so-called global crystal basis of $U^{-}$. Grojnowski and Lusztig [16] proved that the global crystal basis and the canonical basis are the same. The canonical basis $\mathbf{B}$ of $U^{-}$gives rise to bases in all irreducible integrable $U$-representations. Lusztig 34] also produced an idempotent version $\dot{U}$ of $U$ and defined a basis there.

The work of Ariki [1] can be viewed as a categorification of the restricted dual of $U^{-}(\mathfrak{g})$ for $\mathfrak{g}=\mathfrak{s l}_{N}$ and $\mathfrak{g}=\widehat{\mathfrak{s l}}_{N}$ and a categorification of all irreducible integrable representations of these Lie algebras (see also 27, 2, 3], 37]). An integral version of the restricted dual of $U^{-}(\mathfrak{g})$ becomes the sum of Grothendieck groups of suitable blocks of affine Hecke algebra representations. An earlier work of Zelevinsky 47] can be understood in this context as a parametrization of basis elements of $U^{-}(\mathfrak{g})^{*}$ via certain irreducible representations of affine Hecke algebras. Irreducible integrable representations of $U(\mathfrak{g})$ become Grothendieck groups of Ariki-Koike cyclotomic Hecke algebras, which are certain finite-dimensional quotient algebras of affine Hecke algebras.

Grojnowski 14 found a purely algebraic way to understand these categorifications via a generalization of Kleshchev's methods for studying modular representations of the symmetric group [22, 23], 24]. This approach was further developed by Grojnowski and Vazirani [17, Vazirani [45, [46, Brundan and Kleshchev [8]

Received by the editors August 7, 2008.

2000 Mathematics Subject Classification. Primary 81R50; 16 S99.

Key words and phrases. Categorification, quantum groups, Grothendieck ring, canonical basis.

(C)2009 American Mathematical Society Reverts to public domain 28 years from publication 
and others. It is explained by Kleshchev in [25] in the context of degenerate affine Hecke algebras.

In this paper we introduce graded algebras categorifying $U_{q}^{-}(\mathfrak{g})$, for an arbitrary simply-laced $\mathfrak{g}$. We start with an unoriented graph $\Gamma$ without loops and multiple edges. Let $I$ be the set of vertices of $\Gamma$. The bilinear Cartan form on $\mathbb{N}[I]$ is given on the basis elements $i, j \in I$ by

$$
i \cdot j= \begin{cases}2 & \text { if } i=j, \\ -1 & \text { if } i \text { and } j \text { are joined by an edge, } \\ 0 & \text { otherwise. }\end{cases}
$$

The algebra $U^{-}$over $\mathbb{Q}(q)$, the negative (or positive) half of the quantum universal enveloping algebra, has generators $\theta_{i}, i \in I$, and defining relations

$$
\begin{aligned}
\theta_{i} \theta_{j} & =\theta_{j} \theta_{i} \text { if } i \cdot j=0, \\
\left(q+q^{-1}\right) \theta_{i} \theta_{j} \theta_{i} & =\theta_{i}^{2} \theta_{j}+\theta_{j} \theta_{i}^{2} \text { if } i \cdot j=-1 .
\end{aligned}
$$

The algebra $U^{-}$contains a subring ${ }_{\mathcal{A}} \mathbf{f}$, which is the $\mathbb{Z}\left[q, q^{-1}\right]$-lattice generated by all products of quantum divided powers $\theta_{i}^{(a)}$. The canonical basis $\mathbf{B}$ is a basis of ${ }_{\mathcal{A}} \mathbf{f}$ viewed as a free $\mathbb{Z}\left[q, q^{-1}\right]$-module.

In Section 2 of this paper to each graph $\Gamma$ as above we assign a family of graded rings $R(\nu)$, over $\nu \in \mathbb{N}[I]$. The rings are defined geometrically, via braid-like plane diagrams which consist of interacting strings labelled by vertices of the graph. We prove basic results about these rings, then switch from the ground ring $\mathbb{Z}$ to a field $\mathbb{k}$ to simplify the study of $R(\nu)$-modules. We show that the representation theory of $R(\nu)$ categorifies the integral form ${ }_{\mathcal{A}} \mathbf{f}$ of $U^{-}$. We consider the category $R(\nu)$-pmod of finitely-generated graded left projective $R(\nu)$-modules and its Grothendieck group $K_{0}(R(\nu))$. Let $R=\bigoplus_{\nu} R(\nu)$ and define

$$
K_{0}(R)=\bigoplus_{\nu \in \mathbb{N}[I]} K_{0}(R(\nu)) .
$$

Induction and restriction functors coming from the inclusions $R(\nu) \otimes R\left(\nu^{\prime}\right) \subset R(\nu+$ $\left.\nu^{\prime}\right)$ give rise to the multiplication and comultiplication homomorphisms

$$
K_{0}(R) \otimes K_{0}(R) \longrightarrow K_{0}(R), \quad K_{0}(R) \longrightarrow K_{0}(R) \otimes K_{0}(R),
$$

which satisfy the same properties as those for ${ }_{\mathcal{A}} \mathbf{f}$. We define a homomorphism of $\mathbb{Z}\left[q, q^{-1}\right]$-algebras $\gamma:{ }_{\mathcal{A}} \mathbf{f} \longrightarrow K_{0}(R)$ that also respects comultpiplication and takes a divided powers product element $\theta=\theta_{i_{1}}^{\left(a_{1}\right)} \ldots \theta_{i_{r}}^{\left(a_{r}\right)}$ to the image of a certain projective module $P_{\theta}$ in the Grothendieck group.

The quantum Gabber-Kac theorem implies that $\gamma$ is injective. By mirroring for the case of rings $R(\nu)$ the methods of Kleshchev, Grojnowski, and Vazirani, who studied socles of induction and restriction applied to irreducible representations, we show that the homomorphism $\gamma$ is surjective for any graph $\Gamma$ and any field $\mathbb{k}$. The main result of the paper is the following theorem.

Theorem 1.1. $\gamma:{ }_{\mathcal{A}} \mathbf{f} \longrightarrow K_{0}(R)$ is an isomorphism of $\mathbb{N}[I]$-graded twisted bialgebras.

The term "twisted bialgebras" is used above, since the comultiplication in ${ }_{\mathcal{A}} \mathbf{f}$ and $K_{0}(R)$ becomes an algebra homomorphism only after the multiplication in the tensor squares ${ }_{\mathcal{A}} \mathbf{f}^{\otimes 2}$ and $K_{0}(R)^{\otimes 2}$ is twisted by powers of $q$. 
We conjecture that, when $\Gamma$ is a tree and $\mathbb{k}=\mathbb{C}$, the isomorphism $\gamma$ takes canonical basis elements to the images of indecomposable projective modules in $K_{0}(R)$. When the graph is a single vertex, this conjecture is almost trivial. We verify the conjecture in a simple case of the graph $\Gamma$ with two vertices and one edge, with all canonical basis elements being monomials.

The rings $R(\nu)$ should be linked to Lusztig's geometric realization of ${ }_{\mathcal{A}} \mathbf{f}$ :

Conjecture 1.2. If $\mathbb{k}=\mathbb{C}$ and graph $\Gamma$ is a tree, the algebra $R(\nu)$ is Morita equivalent to the algebra of equivariant ext groups $\operatorname{Ext}_{G_{\nu}}(L, L)$, where $G_{\nu}=\prod_{i \in I} G L\left(\nu_{i}\right)$ and $L$ is the sum of simple perverse sheaves $L_{b}$, over all $b \in \mathbf{B}_{\nu}$, in Lusztig's geometric realization of ${ }_{\mathcal{A}} \mathbf{f}$.

This conjecture should follow from an isomorphism between $R(\nu)$ and a suitable convolution algebra. When $\Gamma$ contains cycles, it is possible to modify $R(\nu)$ by introducing "monodromies" around the cycles, and the conjecture is likely to hold for a modified version of $R(\nu)$.

Our results hint at the relation between representation theory of affine Hecke algebras for $G L(n)$ when $q$ is not a root of unity and representations of $R(\nu)$ when the graph $\Gamma$ is a chain. We conjecture that completions of affine Hecke algebras along suitable maximal central ideals are Morita equivalent (or even isomorphic) to completions of $R(\nu)$ along the grading. The above conjectures, if true, would link Lusztig's geometrization of $U^{-}$with Ariki's categorification of $U^{-}$and its restricted dual for $q=1$ and $\Gamma=A_{n}$.

We arrived at the definition of rings $R(\nu)$ from computations involving homomorphisms of bimodules over cohomology rings of partial flag varieties. The bimodules themselves are the cohomology groups of partial and iterated flag varieties that give correspondences for the action of generators $e_{i}$ and $f_{i}$ of quantum $\mathfrak{s l}_{N}$ in the Beilinson-Lusztig-MacPherson geometric model [4] of quantum $\mathfrak{s l}_{N}$. This model was given a categorical interpretation by Grojnowski and Lusztig [15] and later reinterpreted, for $N=2$, via translation and Zuckerman functors in [6], with various generalizations constructed in [13, 44, 48, and in a very recent striking work 49 .

In Section 3.4 we define certain quotient algebras of $R(\nu)$ and conjecture that their categories of modules categorify irreducible integrable representations of $U_{q}(\mathfrak{g})$. A straightforward generalization of our constructions and results from algebras $R(\nu)$ and their quotient algebras in the simply-laced case to that of an arbitrary symmetrizable Kac-Moody algebra $\mathfrak{g}$ will be presented in a follow-up paper.

In the paper [9] Chuang and Rouquier defined $s l(2)$-categorifications, substantiated them with many diverse examples, and applied them to the modular representation theory. Partially inspired by [9] and [10], the second author suggested and investigated a categorification of Lusztig's idempotent completion $\dot{U}$ of quantum $s l(2)$ in [28, 29]. A definition of a categorification of $\dot{U}_{q}(\mathfrak{g})$ for any simply-laced $\mathfrak{g}$ can be obtained by combining the diagrammatic relations of $R(\nu)$ with those of Ü-categorification in 28 . 
In his recent talk, R. Rouquier 42] defined $s l(N)$ and affine $s l(N)$-categorifications and outlined a conjectural program that aims to vastly generalize his prior work with J. Chuang [9] on $s l(2)$-categorifications. We expect Rouquier's and our approaches to be closely related. $\mathrm{R}$. Rouquier informed us that a signed version of rings $R(\nu)$ appears in his categorification [43] of $U(\mathfrak{g})$ for a simply-laced $\mathfrak{g}$.

\section{Rings $R(\nu)$ AND THEIR PROPERTIES}

2.1. Definitions. We fix a graph $\Gamma$, not necessarily finite, with a set of vertices $I$ and unoriented edges $E_{\Gamma}$. We require that $\Gamma$ has no loops and multiple edges. By $\mathbb{N}[I]$ we denote the commutative semigroup freely generated by vertices of $\Gamma$ and for $\nu \in \mathbb{N}[I]$ we write

$$
\nu=\sum_{i \in I} \nu_{i} \cdot i, \quad \nu_{i} \in \mathbb{N}, \quad \mathbb{N}=\{0,1,2, \ldots\} .
$$

Let $|\nu|=\sum \nu_{i} \in \mathbb{N}$, and $\operatorname{Supp}(\nu)=\left\{i \mid \nu_{i} \neq 0\right\}$. We define a bilinear form on $\mathbb{Z}[I]$ by $i \cdot i=2, i \cdot j=-1$ if $i$ and $j$ are connected by an edge, and $i \cdot j=0$ otherwise. In the basis $\{i\}_{i \in I}$ of vertices the bilinear form is given by the Cartan matrix of $\Gamma$.

To $\Gamma$ we associate a diagrammatic calculus of planar diagrams. We consider collections of arcs on the plane connecting $m$ points on one horizontal line with $m$ points on another horizontal line. The position of $m$ points on the line is fixed once and for all (for instance, we could take points $\{1,2, \ldots, m\} \in \mathbb{R}$ ). Arcs have no critical points when projected to the $y$-axis of the plane (a condition reminiscent of braids). Each arc is labelled by a vertex of $\Gamma$. Arcs can intersect, but no triple intersections are allowed. An arc can carry dots. An example of such a diagram is

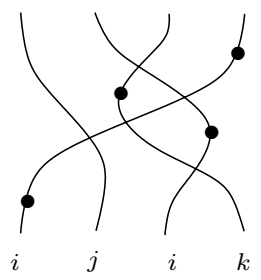

where $i, j, k$ are vertices of $\Gamma$ and the label of an arc is written at the bottom of the arc. We allow isotopies that do not change the combinatorial type of the diagram and do not create critical points for the projection onto the $z$-axis:
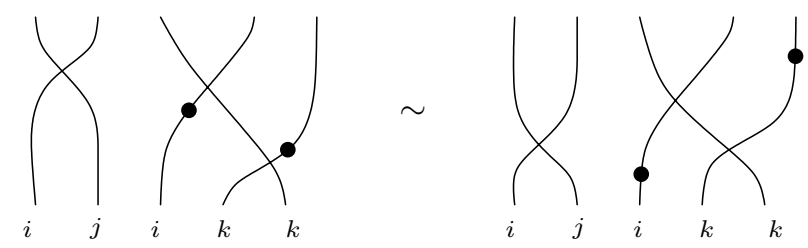
We proceed by allowing finite linear combinations of these diagrams with integral coefficients, modulo the following local relations:
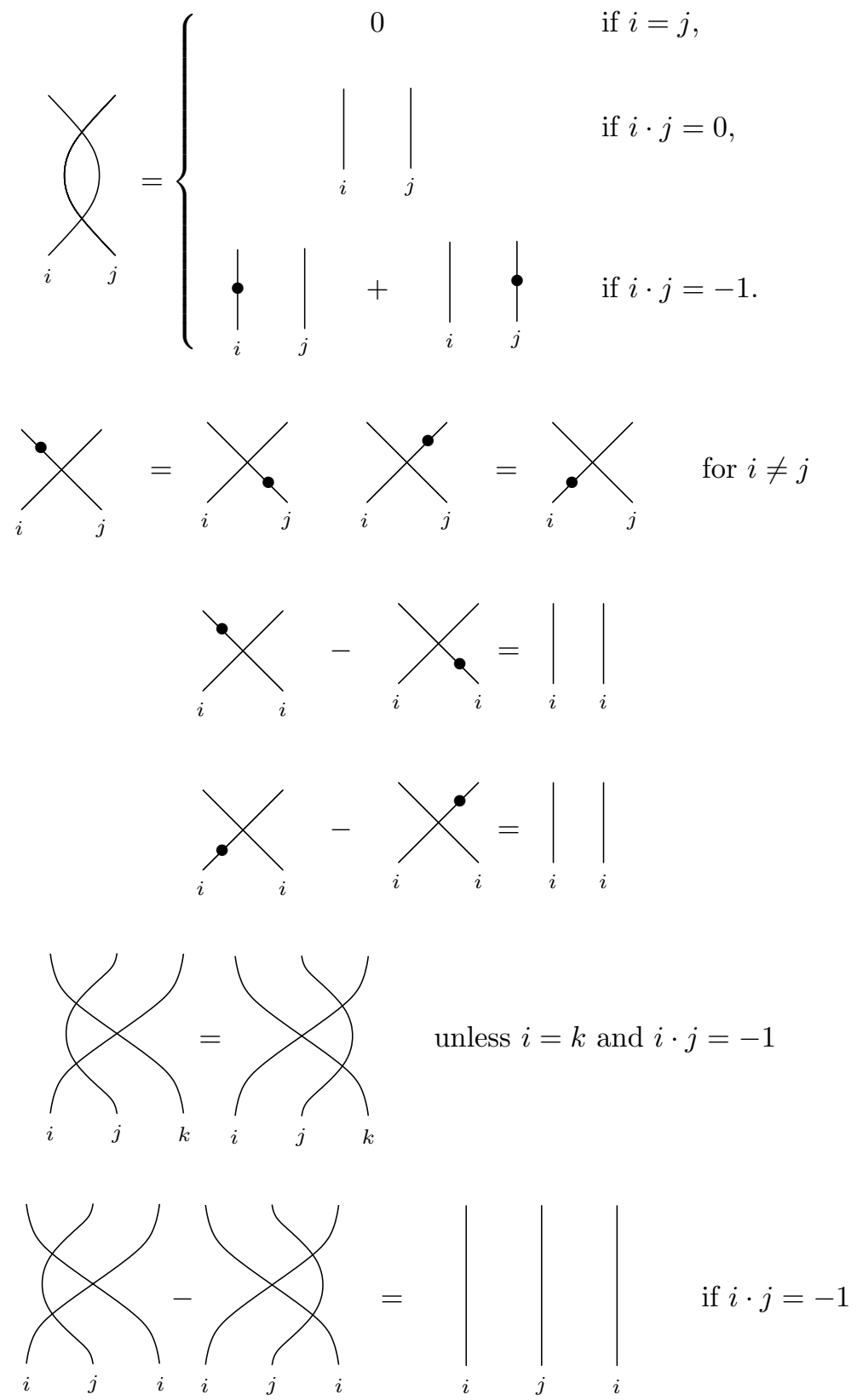

Fix $\nu \in \mathbb{N}[I]$. Let $\operatorname{Seq}(\nu)$ be the set of all sequences of vertices $i=i_{1} \ldots i_{m}$ where $i_{k} \in I$ for each $k$ and vertex $i$ appears $\nu_{i}$ times in the sequence. The length $m$ of the sequence is equal to $|\nu|$ and the cardinality of $\operatorname{Seq}(\nu)$ is equal to $\left(\begin{array}{c}\nu \\ \nu_{i}, \nu_{j}, \ldots\end{array}\right)$, taken over all $i \in I$. For instance,

$$
\operatorname{Seq}(2 i+j)=\{i i j, i j i, j i i\}
$$


Each diagram $D$ as described above determines the two sequences $\operatorname{bot}(D)$ and $\operatorname{top}(D)$ in $\operatorname{Seq}(\nu)$ for some $\nu$. The sequence $\operatorname{bot}(D)$ is given by reading the labels of arcs of $D$ at the bottom position from left to right. We define top $(D)$ likewise. For instance, for the diagram in (2.2), $\operatorname{bot}(D)=i j i k$ and $\operatorname{top}(D)=j i k i$. We often abbreviate sequences with many equal consecutive terms, and write $i_{1}^{n_{1}} \ldots i_{r}^{n_{r}}$ for $i_{1} \ldots i_{1} i_{2} \ldots i_{2} \ldots i_{r} \ldots i_{r}$, where $n_{1}+\cdots+n_{r}=m$.

Define the ring $R(\nu)$ as follows:

$$
R(\nu)=\bigoplus_{i, j \in \operatorname{Seq}(\nu)}{ }_{j} R(\nu)_{i}
$$

as an abelian group, where ${ }_{j} R(\nu)_{i}$ is the abelian group of all linear combinations of diagrams with $\operatorname{bot}(D)=\boldsymbol{i}$ and $\operatorname{top}(D)=\boldsymbol{j}$ modulo the relations (2.3)-(2.7). The product in $R(\nu)$ is given by concatenation (see the left diagram in (2.11) below),

$$
{ }_{k} R(\nu)_{j} \otimes{ }_{j} R(\nu)_{i} \rightarrow{ }_{k} R(\nu)_{i},
$$

and $x y=0$ for $x \in{ }_{l} R(\nu)_{k}$ and $y \in{ }_{j} R(\nu)_{i}$ if $\boldsymbol{k} \neq \boldsymbol{j}$.
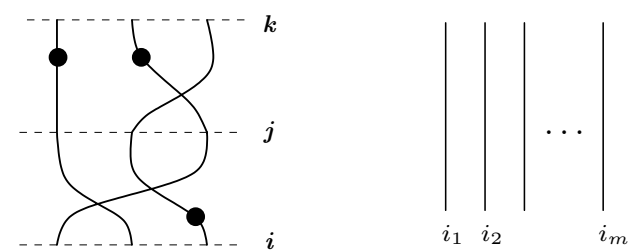

By construction, $R(\nu)$ is an associative ring. For each $i \in \operatorname{Seq}(\nu)$ the diagram $1_{i} \in{ }_{i} R(\nu)_{i}$ shown on the right of (2.11) is an idempotent, $1_{i}^{2}=1_{i}, x 1_{i}=x$ for all $x \in{ }_{j} R(\nu)_{i}$ and $1_{i} x=x$ for all $x \in{ }_{i} R(\nu)_{j}$, for all $\boldsymbol{j}$. Furthermore, $1=\sum_{i \in \operatorname{Seq}(\nu)} 1_{i}$ is the unit element of $R(\nu)$. We turn $R(\nu)$ into a graded ring by declaring degrees of the generators to be

$$
\operatorname{deg}\left(\oint_{i}\right)=2, \quad \operatorname{deg}\left(\searrow_{i}\right)=-i \cdot j .
$$

Let

$$
P_{i}=\bigoplus_{j \in \operatorname{Seq}(\nu)}{ }_{j} R(\nu)_{i}, \quad{ }_{j} P=\bigoplus_{i \in \operatorname{Seq}(\nu)}{ }_{j} R(\nu)_{i} .
$$

$P_{i}$ is a left graded projective $R(\nu)$-module and ${ }_{j} P$ is a right graded projective $R(\nu)$-module.

Flipping a diagram on a horizontal axis induces a grading-preserving anti-involution $\psi$ of $R(\nu)$ which takes ${ }_{j} R(\nu)_{i}$ to ${ }_{i} R(\nu)_{j}$ and $1_{i}$ to $1_{i}$. Flipping a diagram on a vertical axis and simultaneously taking

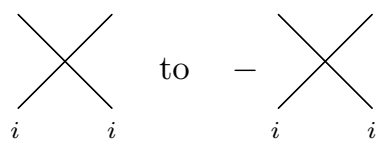

(in other words, multiplying the diagram by $(-1)^{s}$ where $s$ is the number of times equally labelled strands intersect) is an involution $\sigma$ of $R(\nu)$ which commutes with $\psi$. 
Sometimes it is convenient to convert from graphical to algebraic notation. For a sequence $\boldsymbol{i}=i_{1} i_{2} \ldots i_{m} \in \operatorname{Seq}(\nu)$ and $1 \leq k \leq m$ we denote

$$
x_{k, i}:=\left.\left.\left.\right|_{i_{1}} \cdots\right|_{i_{k}} \cdots\right|_{i_{m}}
$$

with the dot positioned on the $k$-th strand counting from the left, and

$$
\delta_{k, i}:=\left.||_{i_{1}} \cdots \searrow_{i_{k}} \cdots\right|_{i_{k+1}} \ldots
$$

The symmetric group $S_{m}$ acts on $\operatorname{Seq}(\nu), m=|\nu|$ by permutations. Transposition $s_{k}=(k, k+1)$ switches entries $i_{k}, i_{k+1}$ of $\boldsymbol{i}$. Thus, $\delta_{k, i} \in s_{k}(i) R(\nu)_{i}$. The relations (2.3) become

$$
\delta_{k, s_{k}(i)} \delta_{k, i}=\left\{\begin{array}{ccc}
0 & \text { if } & i_{k}=i_{k+1} \\
1_{i} & \text { if } & i_{k} \cdot i_{k+1}=0 \\
x_{k, i}+x_{k+1, i} & \text { if } & i_{k} \cdot i_{k+1}=-1
\end{array}\right.
$$

Other defining relations for $R(\nu)$ can be similarly rewritten.

\subsection{Examples.}

1) $\nu=0$. We have $R(0)=\mathbb{Z}$, with the unit element given by the empty diagram.

2) $\nu=i$ for some vertex $i$. Then a diagram is a line with some number of dots on

it. Hence, $R(i) \cong \mathbb{Z}\left[x_{1, i}\right]$, where in our notation $x_{1, i}$ denotes a line labelled $i$ with one dot on it.

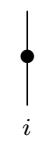

3) $\nu=m i$ for some vertex $i$. The only sequence in $\operatorname{Seq}(m i)$ is $i^{m}=i i \ldots i$. Every strand in the diagram is labelled by $i$, and the local relations are

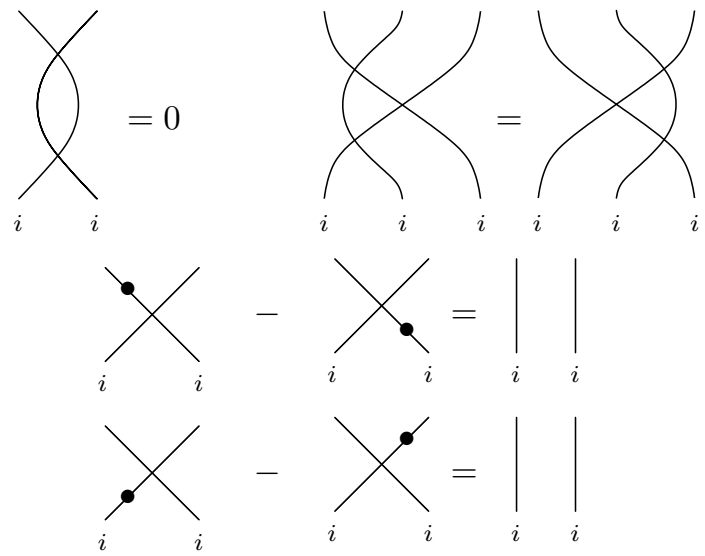

$R(m i)$ is isomorphic to the nil-Hecke ring $\mathrm{NH}_{m}$, which is the unital ring of endomorphisms of the abelian group $\mathbb{Z}\left[x_{1}, \ldots, x_{m}\right]$ generated by the endomorphisms of multiplication by $x_{1}, \ldots, x_{m}$ and the divided difference operators

$$
\partial_{a}(f(x))=\frac{f(x)-s_{a} f(x)}{x_{a}-x_{a+1}}, \quad 1 \leq a \leq m-1,
$$


where $s_{a}$ transposes $x_{a}$ and $x_{a+1}$ in the polynomial $f(x)$. The defining relations are

$$
\begin{aligned}
& x_{a} x_{b}=x_{b} x_{a}, \\
& \partial_{a} x_{b}=x_{b} \partial_{a} \quad \text { if }|a-b|>1, \quad \partial_{a} \partial_{b}=\partial_{b} \partial_{a} \quad \text { if }|a-b|>1, \\
& \partial_{a}^{2}=0, \quad \partial_{a} \partial_{a+1} \partial_{a}=\partial_{a+1} \partial_{a} \partial_{a+1}, \\
& x_{a} \partial_{a}-\partial_{a} x_{a+1}=1, \quad \partial_{a} x_{a}-x_{a+1} \partial_{a}=1 .
\end{aligned}
$$

In the above equations $x_{a}$ stands for the operator of multiplication by $x_{a}$. The defining relations are exactly our graphical equations on identically-colored strands, with the relations in the first two rows corresponding to isotopies of diagrams.

The nil-Hecke ring is related to the theory of Schubert varieties; see [26], [7. The nil-Coxeter ring is the subring of $N H_{m}$ generated by $\partial_{1}, \ldots, \partial_{m-1}$; see [39, Chapter 2], 21]. Divided differences go back to Newton; in the context of representation theory they appeared in [5], [11.

The center of $N H_{m}$ is the ring of symmetric polynomials in $x_{1}, \ldots, x_{m}$, and $N H_{m}$ is isomorphic to the ring of $m ! \times m !$ matrices with coefficients in $Z\left(N H_{m}\right)$, see 36. Here we consider $N H_{m}$ as a graded ring, with $\operatorname{deg}\left(\partial_{a}\right)=-2$ and $\operatorname{deg}\left(x_{a}\right)=2$. The graded nil-Hecke ring plays a fundamental role in the categorification of Lusztig's quantum $\mathfrak{s l}_{2}$ defined by the second author [28].

For each permutation $w \in S_{m}$ let $\partial_{w}=\partial_{a_{1}} \ldots \partial_{a_{r}}$, where $s_{a_{1}} \ldots s_{a_{r}}$ is a minimal presentation of $w$, so that $r=l(w)$. This element does not depend on the choice of presentation.

Define $e_{m}=x_{1}^{m-1} x_{2}^{m-2} \ldots x_{m-1} \partial_{w_{0}}$, where $w_{0}$ is the longest permutation. This element is an idempotent of degree 0 . We will also use the idempotent $\psi\left(e_{m}\right)$ given by reflecting the diagram of $e_{m}$ on the horizontal axis,

$$
\psi\left(e_{m}\right)=\partial_{w_{0}} x_{1}^{m-1} x_{2}^{m-2} \ldots x_{m-1}
$$

$N H_{m} \psi\left(e_{m}\right)$ is a left $N H_{m}$-module isomorphic to the polynomial representation of $\mathrm{NH}_{m}$. The polynomial representation is, up to isomorphism and grading shifts, the unique graded indecomposable projective $\mathrm{NH}_{m}$-module. The module $N H_{m} \psi\left(e_{m}\right)$ is nontrivial in even nonnegative degrees only.

The regular representation of $N H_{m}$ decomposes as the sum of $m$ ! copies of the polynomial representation. Taking the grading into account and denoting by $P_{m}$ the module $N H_{m} \psi\left(e_{m}\right)$ with the grading shifted down by $\frac{m(m-1)}{2}$, we get a direct sum decomposition of graded modules,

$$
N H_{m} \cong P_{m}^{[m] !}
$$

Here $[m] !=[m][m-1] \ldots[1]$ is the quantum factorial, $[m]=\frac{q^{m}-q^{-m}}{q-q^{-1}}$, and $M^{f}$ or $M^{\oplus f}$, for a graded module $M$ and a Laurent polynomial $f=\sum f_{a} q^{a} \in \mathbb{Z}\left[q, q^{-1}\right]$, denotes the direct sum over $a \in \mathbb{Z}$, of $f_{a}$ copies of $M\{a\}$.

We denote by $P_{i, m}$ the corresponding indecomposable graded projective module over $R(m i)$ and by $e_{i, m}$ the idempotent corresponding to $e_{m}$ under the isomorphism $R(m i) \cong N H_{m}$. As a graded abelian group, $P_{i, m}$ is nontrivial in degrees $-\frac{m(m-1)}{2}+2 \mathbb{N}$. 
Letting ${ }_{m} P$ be the right graded projective module $e_{m} N H_{m}\left\{-\frac{m(m-1)}{2}\right\}$, we have a decomposition of graded right $\mathrm{NH}_{m}$-modules

$$
N H_{m} \cong{ }_{m} P^{[m] !} .
$$

We denote by ${ }_{i, m} P$ the corresponding indecomposable graded projective right $R(m i)$-module. Idempotents $e_{i, m}$ and $\psi\left(e_{i, m}\right)$ have the following diagrammatic presentation for $m=3$ :

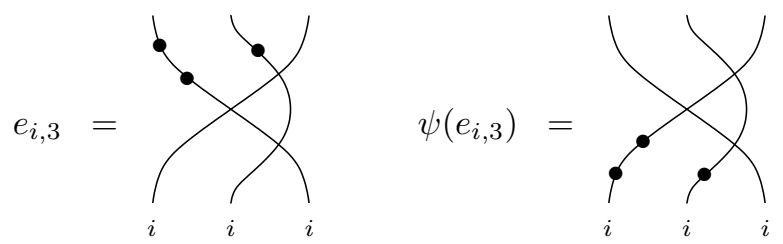

The quotient of $\mathbb{Z}\left[x_{1}, \ldots, x_{m}\right]$ by the ideal of symmetric polynomials is a representation $L_{m}$ of $N H_{m}$ which becomes irreducible upon tensoring with any field $\mathbb{k}$. Over $\mathbb{k}$, any graded irreducible representation of $N H_{m}$ is isomorphic to $L_{m}$, up to a grading shift. We denote the corresponding irreducible representation of $R(m i)$ by $L\left(i^{m}\right)$. It is nonzero in degrees $0,2, \ldots, \frac{m(m-1)}{2}$. The representation $L\left(i^{m}\right)$ is isomorphic to the representation induced from the one-dimensional graded module $L$ over $\mathbb{k}\left[x_{1}, \ldots, x_{m}\right]$ (on which $x_{1}, \ldots, x_{m}$ necessarily act trivially).

Lemma 2.1. The common 0-eigenspace of operators $x_{1}, \ldots, x_{m}$ on $L\left(i^{m}\right) \cong$ $\operatorname{Ind}(L)$ is exactly $1 \otimes L$. All Jordan blocks of $x_{m}$ on $L\left(i^{m}\right)$ are of size $m$.

Proof. Due to the uniqueness of the irreducible module $L\left(i^{m}\right)$, it is isomorphic to the module induced from the trivial representation of the subring of $N H_{m}$ generated by the divided differences $\partial_{1}, \ldots, \partial_{m}$ and symmetric polynomials in $x_{1}, \ldots, x_{m}$. This induced representation is isomorphic, as a $\mathbb{k}\left[x_{1}, \ldots, x_{m}\right]$ module, to the quotient of $\mathbb{k}\left[x_{1}, \ldots, x_{m}\right]$ by the ideal generated by symmetric polynomials without the constant term, and to the cohomology ring of the full flag variety. The lemma follows from the standard facts of this quotient ring.

4) $\nu=i+j$ and $i \cdot j=0$. Seq $(i+j)=\{i j, j i\}$. The $\operatorname{ring} R(\nu)$ is isomorphic to the ring of $2 \times 2$ matrices with coefficients in $\mathbb{Z}\left[x_{1}, x_{2}\right]$. The isomorphism is given on generators by

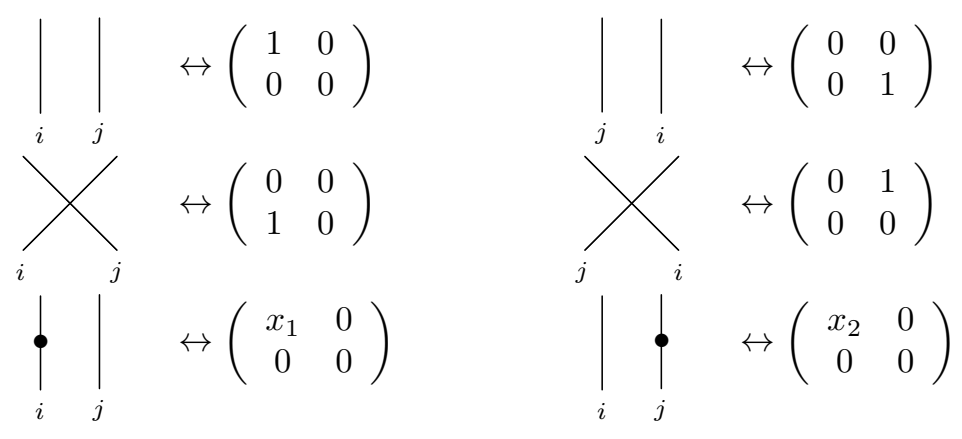


5) $\nu=i_{1}+\cdots+i_{m}$ and $i_{k} \cdot i_{\ell}=0$ for all $k \neq \ell$. Then $R(\nu)$ is isomorphic to the ring of $m ! \times m !$ matrices with coefficients in $\mathbb{Z}\left[x_{1}, \ldots, x_{m}\right]$. To see the isomorphism, enumerate the rows and columns by elements of $\operatorname{Seq}(\nu)$ and send the element ${ }_{j} 1_{i}$ to the elementary $(\boldsymbol{j}, \boldsymbol{i})$-matrix.

6) $\nu=\nu^{\prime}+\nu^{\prime \prime} \operatorname{such}$ that $i \cdot j=0$ for any $i \in \operatorname{Supp}\left(\nu^{\prime}\right)$ and $j \in \operatorname{Supp}\left(\nu^{\prime \prime}\right)$. In this case $R(\nu)$ is isomorphic to the matrix algebra of size $\left(\begin{array}{c}|\nu| \\ \left|\nu^{\prime}\right|,\left|\nu^{\prime \prime}\right|\end{array}\right)$ with coefficients in $R\left(\nu^{\prime}\right) \otimes_{\mathbb{Z}} R\left(\nu^{\prime \prime}\right)$. Indeed, except for crossings, there are no interactions between strands from $\nu^{\prime}$ and strands from $\nu^{\prime \prime}$. A pair $i \in \operatorname{Seq}\left(\nu^{\prime}\right), j \in \operatorname{Seq}\left(\nu^{\prime \prime}\right)$ defines the sequence $\boldsymbol{i} \boldsymbol{j} \in \operatorname{Seq}\left(\nu^{\prime}+\nu^{\prime \prime}\right)$ and

$$
{ }_{i j} R_{i j} \cong R\left(\nu^{\prime}\right) \otimes R\left(\nu^{\prime \prime}\right),
$$

since we can pull apart the $i$ and $j$ strands in any diagram $D$ with $\boldsymbol{i} \boldsymbol{j}=\operatorname{top}(D)=$ $\operatorname{bot}(D)$, using that $i_{k} \cdot j_{\ell}=0$, for all $k$ and $\ell$.

7) $\nu=i+j$ and $i \cdot j=-1$. We can identify $R(i+j)$ with the ring of $2 \times 2$ matrices with coefficients in $\mathbb{Z}\left[x_{1}, x_{2}\right]$ such that the bottom left coefficient is divisible by $x_{1}+x_{2}$ :
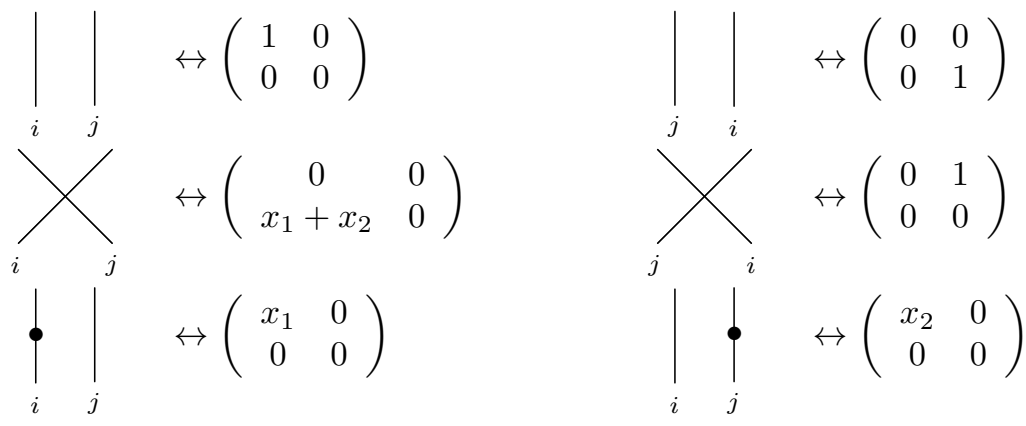

Remark 2.2. If $i \cdot j=-1$, then the elements
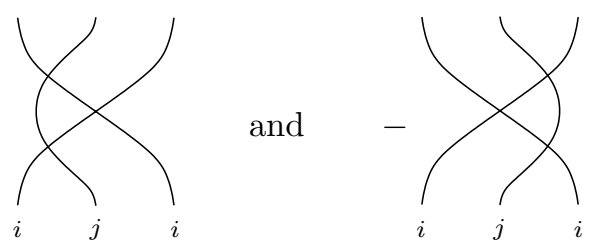

are mutually orthogonal idempotents in $R(2 i+j)$. For instance, 


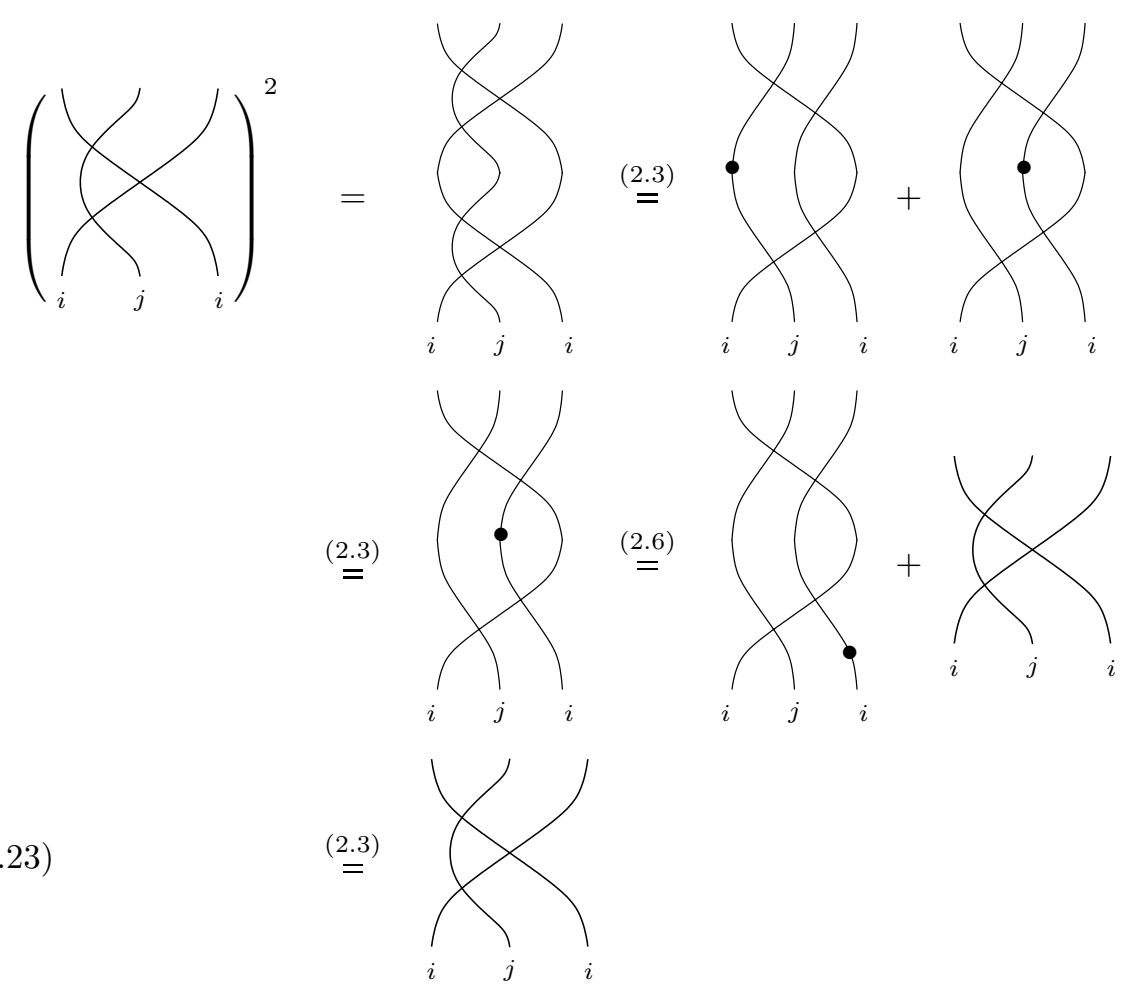

Equation (2.7) can be viewed as a decomposition of the idempotent $1_{i j i}$ into the sum of two orthogonal idempotents. Equation (2.8) can be thought of as allowing triple intersections for certain $i j k$.

2.3. A faithful representation and a basis of $R(\nu)$. Action of $R(\nu)$ on the sum of polynomial spaces. Choose an orientation of each edge of $\Gamma$. For each $\nu \in \mathbb{N}[I]$ we define an action of $R(\nu)$ on the free abelian group

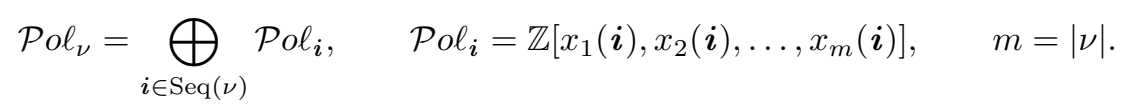

It is useful to think of the variable $x_{k}(\boldsymbol{i})$ as labelled by the vertex of $\Gamma$ in the $k$ th position in the sequence $\boldsymbol{i}$. The symmetric group $S_{m}$ acts on $\mathcal{P} o \ell_{\nu}$ by taking $x_{a}(\boldsymbol{i})$ to $x_{w(a)}(w(\boldsymbol{i})), w \in S_{m}$. The transposition $s_{k}$ maps $x_{a}(\boldsymbol{i})$ to $x_{a}\left(s_{k}(\boldsymbol{i})\right)$ if $a \neq k, k+1, x_{k}(\boldsymbol{i})$ to $x_{k+1}\left(s_{k} \boldsymbol{i}\right)$, and $x_{k+1}(\boldsymbol{i})$ to $x_{k}\left(s_{k} \boldsymbol{i}\right)$.

To define the action of $R(\nu)$, we first require that an element $x \in{ }_{j} R(\nu)_{i}$ acts by 0 on ${\mathcal{P} o \ell_{k}}_{k}$ if $\boldsymbol{k} \neq \boldsymbol{i}$ and takes $\mathcal{P} o \ell_{i}$ to $\mathcal{P} o \ell_{j}$. We describe the action of the generators. The dot in the $k$-th position $x_{k, i}$ (see (2.14)) acts by sending $f \in \mathcal{P}_{0} \ell_{i}$ to $x_{k}(i) f \in \mathcal{P} o \ell_{i}$. The idempotent $1_{i}$ acts by the identity on $\mathcal{P} o \ell_{i}$. The crossing 
$\delta_{k, i}($ see (2.15) $)$ acts on $f \in \mathcal{P}_{o} \ell_{i}$ by

$$
\begin{aligned}
f & \mapsto s_{k} f \quad \text { if } i_{k} \cdot i_{k+1}=0 \\
f & \mapsto \frac{f-s_{k} f}{x_{k}(\boldsymbol{i})-x_{k+1}(\boldsymbol{i})} \text { if } i_{k}=i_{k+1}, \\
f & \mapsto s_{k} f \quad \text { if } i_{k} \longleftarrow i_{k+1}, \\
f & \mapsto\left(x_{k}\left(s_{k} \boldsymbol{i}\right)+x_{k+1}\left(s_{k} \boldsymbol{i}\right)\right)\left(s_{k} f\right) \quad \text { if } \quad i_{k} \longrightarrow i_{k+1} .
\end{aligned}
$$

The notation $i_{k} \longleftarrow i_{k+1}$ means that $i_{k} \cdot i_{k+1}=-1$ and this edge of $\Gamma$ is oriented from $i_{k+1}$ to $i_{k}$. Note that when $i_{k}=i_{k+1}$ the crossing $\delta_{k, i}$ acts by the divided difference operator. When all strands have the same label $i$, the action reduces to the action of the nil-Hecke algebra on its polynomial representation.

Proposition 2.3. These rules define a left action of $R(\nu)$ on $\mathcal{P} o \ell_{\nu}$.

Proof. We check the defining relations for $R(\nu)$. The relation (2.3) with $i \cdot j=0$ and $i \cdot j=-1$ and relation (2.4) are trivial to verify. The relation (2.3) with $i=j$ and relations (2.6), (2.5), and (2.8) for $i=j=k$ are just the nil-Hecke relations. The relation (2.8) with $i, j, k$ all distinct, or with $i \neq j=k, i \cdot j=0$, or with $i=j \neq k, j \cdot k=0$ is easy to check. The same relation with $i=j$, $i \cdot k=-1$ or $j=k, i \cdot j=-1$ follows from the fact that the divided difference operator annihilates symmetric polynomials. This leaves us with the last relation (2.7), reproduced below:

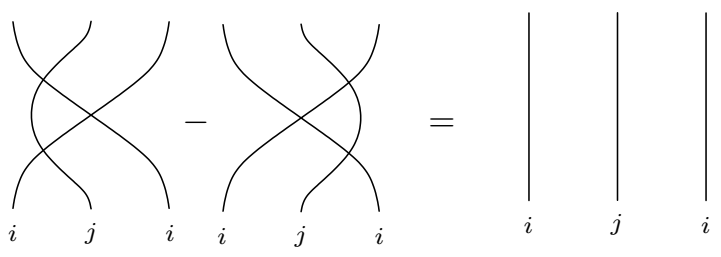

if $i \cdot j=-1$.

It is enough to check it on 3 -stranded diagrams, with $\nu=2 i+j$. To simplify formulas, we write $x, y$, and $z$ instead of $x_{k}(\boldsymbol{i}), x_{k+1}(\boldsymbol{i})$, and $x_{k+2}(\boldsymbol{i})$ for each $\boldsymbol{i}=\{i j i, i i j, j i i\}$. Assume that the $i j$ edge is $i \longleftarrow j$. The left hand side of the relation is

$$
\delta_{1, j i i} \delta_{2, j i i} \delta_{1, i j i}-\delta_{2, i i j} \delta_{1, i i j} \delta_{2, i j i}
$$


taking $\mathcal{P} o \ell_{i j i}$ to $\mathcal{P} o \ell_{i j i}$. We compute the action of this element on each monomial $x^{u} y^{v} z^{w}, u, v, w \in \mathbb{N}$ :

$$
\begin{aligned}
\delta_{1, j i i} & \delta_{2, j i i} \delta_{1, i j i}\left(x^{u} y^{v} z^{w}\right) \\
& =\delta_{1, j i i} \delta_{2, j i i}\left(x^{v} y^{u} z^{w}\right)=\delta_{1, j i i}\left(\frac{x^{v} y^{u} z^{w}-x^{v} y^{w} z^{u}}{y-z}\right) \\
& =\frac{x^{u} y^{v} z^{w}-x^{w} y^{v} z^{u}}{x-z}(x+y), \\
\delta_{2, i i j} & \delta_{1, i i j} \delta_{2, i j i}\left(x^{u} y^{v} z^{w}\right) \\
= & \delta_{2, i i j} \delta_{1, i i j}\left((y+z) x^{u} y^{w} z^{v}\right) \\
= & \delta_{2, i i j}\left(\frac{x^{u} y^{w+1}-x^{w+1} y^{u}}{x-y} z^{v}+\frac{x^{u} y^{w}-x^{w} y^{u}}{x-y} z^{v+1}\right) \\
= & \delta_{2, i i j}\left(\frac{x^{u} y^{w+1} z^{v}-x^{w+1} y^{u} z^{v}+x^{u} y^{w} z^{v+1}-x^{w} y^{u} z^{v+1}}{x-y}\right) \\
= & \frac{x^{u} y^{v} z^{w+1}-x^{w+1} y^{v} z^{u}+x^{u} y^{v+1} z^{w}-x^{w} y^{v+1} z^{u}}{x-z} .
\end{aligned}
$$

One can easily verify that the difference of (2.24) and (2.25) is $x^{u} y^{v} z^{w}$, proving relation (2.7) in this case. When $i \longrightarrow j$, we compute

$$
\begin{aligned}
\delta_{1, j i i} \delta_{2, j i i} \delta_{1, i j i}\left(x^{u} y^{v} z^{w}\right) & \\
& =\delta_{1, j i i} \delta_{2, j i i}\left((x+y) x^{v} y^{u} z^{w}\right) \\
& =\delta_{1, j i i}\left(x^{v+1} \frac{y^{u} z^{w}-y^{w} z^{u}}{y-z}+x^{v} \frac{y^{u+1} z^{w}-y^{w} z^{u+1}}{y-z}\right) \\
& =\delta_{1, j i i}\left(\frac{x^{v+1} y^{u} z^{w}-x^{v+1} y^{w} z^{u}+x^{v} y^{u+1} z^{w}-x^{v} y^{w} z^{u+1}}{y-z}\right) \\
& =\frac{x^{u} y^{v+1} z^{w}-x^{w} y^{v+1} z^{u}+x^{u+1} y^{v} z^{w}-x^{w} y^{v} z^{u+1}}{x-z}, \\
\delta_{2, i i j} \delta_{1, i i j} \delta_{2, i j i}\left(x^{u} y^{v} z^{w}\right) & =\delta_{2, i i j} \delta_{1, i i j}\left(x^{u} y^{w} z^{v}\right) \\
= & \delta_{2, i i j}\left(\frac{x^{u} y^{w} z^{v}-x^{w} y^{u} z^{v}}{x-y}\right) \\
= & \frac{x^{u} y^{v} z^{w}-x^{w} y^{v} z^{u}}{x-z}(y+z) .
\end{aligned}
$$

Again, the difference of (2.26) and (2.27) is $x^{u} y^{v} z^{w}$. Relation (2.7) and Proposition 2.3 follow.

A spanning set. We look for a lower bound on the size of $R(\nu)$. An element of this ring is a linear combination of diagrams.

If a diagram $D$ contains two strands that intersect more than once, relations (2.3) - 2.7) allow us to write $D$ as a linear combination of diagrams, each with fewer intersections than $D$. Iterating, we can write any element of $R(\nu)$ as a linear combination of diagrams with at most one intersection between any two strands. Furthermore, we can slide all dots in a diagram $D$ all the way to the bottom of the diagram at the cost of adding a linear combination of diagrams with fewer crossings than $D$. These two operations together tell us that $R(\nu)$ is spanned by diagrams 
having all dots at the bottom and with each pair of strands intersecting at most once:

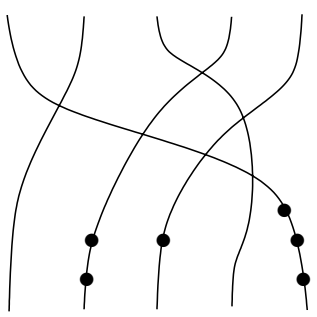

Such $D$ are determined by $i=\operatorname{bot}(D)$, a minimal presentation $\widetilde{w}=s_{k_{1}} \ldots s_{k_{r}}$, $r=l(w)$ of a permutation $w \in S_{m}$ and the number of dots at each bottom endpoint of $D$. The difference of two diagrams given by the same data except for different minimal presentations of $w$ can be written as a linear combination of diagrams with fewer crossings than each of the original two diagrams.

For each $w \in S_{m}$ fix its minimal presentation $\widetilde{w}$. For $\boldsymbol{i}, \boldsymbol{j} \in \operatorname{Seq}(\nu)$ let ${ }_{j} S_{\boldsymbol{i}}$ be the subset of $S_{m}$ consisting of permutations $w$ that take $i$ to $\boldsymbol{j}$ via the standard action of permutations on sequences, defined earlier. For each $w \in{ }_{j} S_{i}$ we convert its minimal presentation $\widetilde{w}$ into an element of ${ }_{j} R(\nu)_{i}$ denoted $\widehat{w}_{i}$. Denote the subset $\left\{\widehat{w}_{i}\right\}_{w \in \in_{j} S_{i}}$ of ${ }_{j} R(\nu)_{i}$ by ${ }_{j} \widehat{S}_{i}$.

Example 2.4. ${ }_{i j i} S_{i j i}=\{\mathrm{id},(13)\}$, and
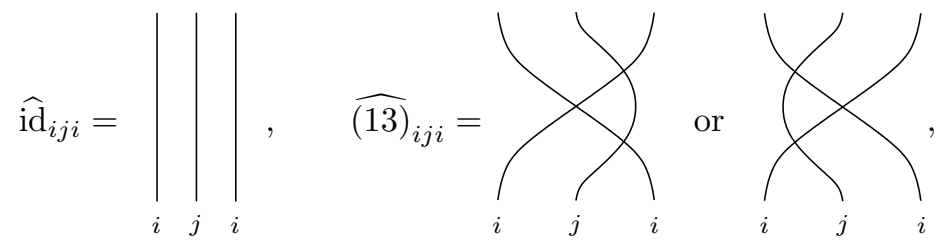

depending on whether we choose $s_{2} s_{1} s_{2}$ or $s_{1} s_{2} s_{1}$ as a minimal presentation of permutation (13).

In general, ${ }_{j} \widehat{S}_{i}$ depends on our choices of minimal presentations for permutations. For instance, in the above example, $\widehat{(13)}_{i j i} \in{ }_{i j i} \widehat{S}_{i j i}$ will depend nontrivially on whether the presentation $s_{1} s_{2} s_{1}$ or $s_{2} s_{1} s_{2}$ was chosen if $i \cdot j=-1$.

Let ${ }_{j} B_{i}$ be the set $\left\{y \cdot x_{1, i}^{u_{1}} \ldots x_{m, i}^{u_{m}}\right\}$ over all $y \in{ }_{j} \widehat{S}_{i}$ and $u_{i} \in \mathbb{N}$. Here the diagrams in ${ }_{j} \widehat{S}_{i}$ are multiplied by all possible monomials at the bottom. For example, the sets ${ }_{i j} B_{i j}$ and ${ }_{j i} B_{i j}$ consist of elements

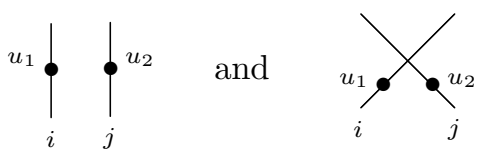

respectively, where we write

$$
{ }^{u} \downarrow=(\downarrow)^{u} .
$$

Theorem 2.5. ${ }_{j} R(\nu)_{i}$ is a free graded abelian group with a homogeneous basis ${ }_{j} B_{i}$. 
Proof. We have already observed that the set ${ }_{j} B_{i}$ spans ${ }_{j} R(\nu)_{i}$. This set consists of homogeneous elements relative to our grading on $R(\nu)$. To prove linear independence of elements of ${ }_{j} B_{i}$ we check that they act on $\mathcal{P} o \ell_{\nu}$ by linearly independent operators.

Let ${ }_{j} 1_{i}$ be the diagram with the fewest number of crossings with $\operatorname{bot}\left({ }_{j} 1_{i}\right)=i$ and $\operatorname{top}\left({ }_{j} 1_{i}\right)=\boldsymbol{j}$. For example,

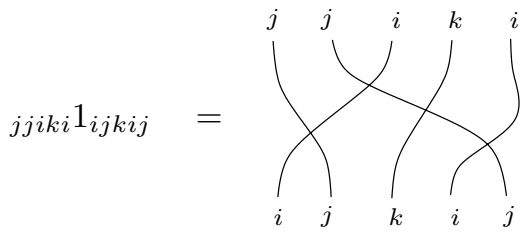

Note that identically colored lines do not intersect in ${ }_{j} 1_{i}$, and that $i 1_{i}$ is just $1_{i}$.

The product ${ }_{i} 1_{j j} 1_{i}=\prod\left(x_{a, i}+x_{b, i}\right)$ where the product is over all pairs $1 \leq a<$ $b \leq m$ such that the lines in ${ }_{j} 1_{i}$ ending at $a$ and $b$ bottom endpoints counting from the left intersect and are colored by $i, j$ with $i \cdot j=-1$.

For instance, if $\boldsymbol{i}=i j j, \boldsymbol{j}=j j i$ with $i \cdot j=-1$, then

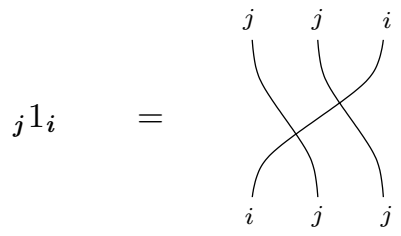

and the product

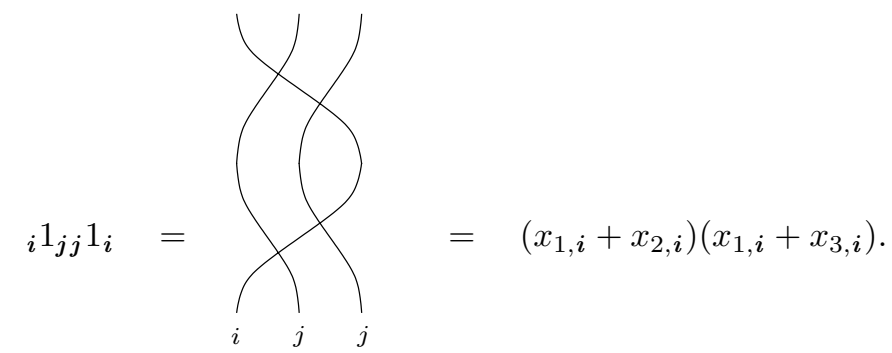

Choose a complete order on the set of vertices of $\Gamma$ and orient $\Gamma$ so that for each edge $i \longrightarrow j$ we have $i<j$ relative to the order. This order induces a lexicographic order on Seq $(\nu)$. We prove linear independence of ${ }_{j} B_{i}$ by induction on $j \in \operatorname{Seq}(\nu)$ with respect to this order.

Base of induction: We write

$$
\boldsymbol{j}=j_{1} \ldots j_{1} j_{2} \ldots j_{2} \ldots j_{r} \ldots j_{r}=j_{1}^{\nu_{1}} j_{2}^{\nu_{2}} \ldots j_{r}^{\nu_{r}} \in \operatorname{Seq}(\nu), \quad \nu=\sum_{k=1}^{r} \nu_{k} j_{k}
$$

where $j_{1}<j_{2}<\cdots<j_{r}$ and $r$ is the cardinality of $\operatorname{Supp}(\nu)$. Clearly, $\boldsymbol{j}$ is the lowest element in $\operatorname{Seq}(\nu)$ with respect to lexicographic order. For this $j$ each $w \in{ }_{j} S_{i}$ can be written uniquely as $w=w_{1} w_{0}$ where $w_{1} \in S_{\nu_{1}} \times \cdots \times S_{\nu_{r}}$ and $w_{0}$ is the unique minimal length element in ${ }_{j} S_{i}$.

Each minimal length representative $\widetilde{w_{0}}$ determines the same ${\widehat{w_{0}}}_{i}={ }_{j} 1_{i}$. Likewise, the element $\widehat{w}_{1 j}$ does not depend on the choice of a minimal length representative 
$\widetilde{w_{1}}$, since in the nil-Hecke algebra the element associated to a permutation does not depend on the minimal presentation of this permutation.

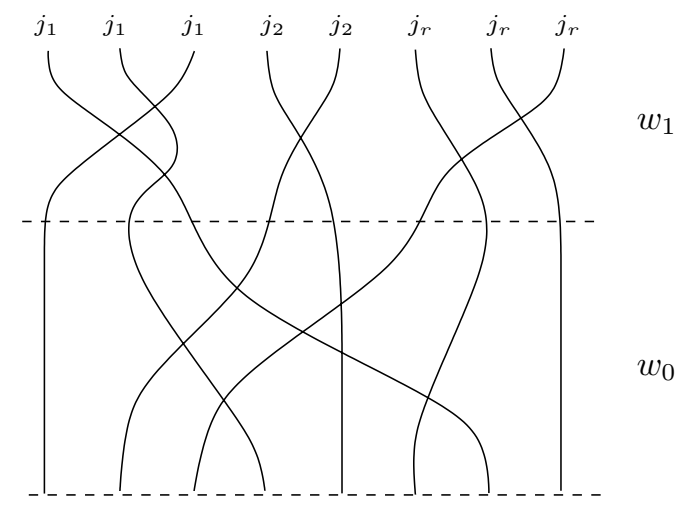

The set ${ }_{j} B_{i}$ consists of elements $\widehat{w}_{1 j}{\widehat{w_{0}}}_{i} x^{u}$, over all $u \in \mathbb{N}^{m}$, where

$$
x^{u}=x_{1, i}^{u_{1}} x_{2, i}^{u_{2}} \ldots x_{m, i}^{u_{m}} .
$$

It suffices to check that induced maps

$$
\widehat{w}_{1 j}{\widehat{w_{0}}}_{i} x^{u}:{\mathcal{P} o \ell_{i}}^{\longrightarrow} \mathcal{P} o \ell_{j}
$$

are linearly independent, over all $u \in \mathbb{N}^{m}$. Indeed, ${\widehat{w_{0}}}_{i} x^{u}$ takes $x^{v} \in \mathcal{P} o \ell_{i}$ to $x^{w_{0}(u+v)} \in \mathcal{P}_{o} \ell_{j}$, where $w_{0}$ acts on $v$ via the obvious permutation. This is due to peculiarities of our action, since the element $\delta_{k, i}$ takes $f \in \mathcal{P} o \ell_{i}$ to $s_{k} f$ if $i_{k}>i_{k+1}$. Elements $\widehat{w_{1}}$ act on the monomials by products of divided difference operators. It is known that the standard action of the nil-Hecke ring on polynomials is faithful [36], implying linear independence of all maps (2.31).

Induction step: Assume we proved that ${ }_{j} B_{i}$ is independent, that $j_{k}<j_{k+1}$, and set $\boldsymbol{j}^{\prime}=s_{k} \boldsymbol{j}=j_{1} \ldots j_{k-1} j_{k+1} j_{k} j_{k+2} \ldots j_{m}$. It suffices to assume that $j_{k} \cdot j_{k+1}=$ -1 , otherwise $j_{k} \cdot j_{k+1}=0$ and the maps $\delta_{k, j}, \delta_{k, j^{\prime}}$ set up bijections between ${ }_{j} B_{i}$ and $j^{\prime} B_{i}$, implying linear independence of $j^{\prime} B_{i}$.

To show that $j^{\prime} B_{i}$ is independent, we examine its image under the map

$$
\delta_{k, j^{\prime}}: j^{\prime} R(\nu)_{i} \rightarrow{ }_{j} R(\nu)_{i} .
$$

Define a partial order on ${ }_{j} B_{i}$ by requiring that $w_{1} x^{u}<w_{2} x^{v}$ if $\ell\left(w_{1}\right)<\ell\left(w_{2}\right)$, or if $w_{1}=w_{2}, u_{1}=v_{1}, \ldots, u_{t}=v_{t}, u_{t+1}<v_{t+1}$ for some $t$. Extend this partial order to a complete order on ${ }_{j} B_{i}$ in some way.

Define the map $\bar{\delta}:{ }_{j^{\prime}} B_{i} \rightarrow{ }_{j} B_{i}$ by $\bar{\delta} y=\delta_{k, j^{\prime}} y$ if the strands of diagram $y$ ending at the top endpoints numbered $k, k+1$ from the left are disjoint

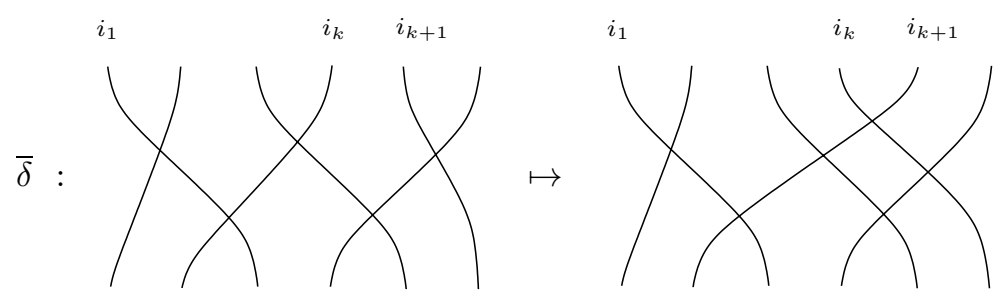

and $\bar{\delta} y=y^{\prime} \cdot x_{\ell, i}$ if these two strands of $y$ intersect. Here $\ell$ is the number, counting from the left, of the bottom endpoint of the strand with top endpoint $k$, and $y^{\prime}$ is 
obtained from $y$ by removing the intersection of these two strands. Graphically

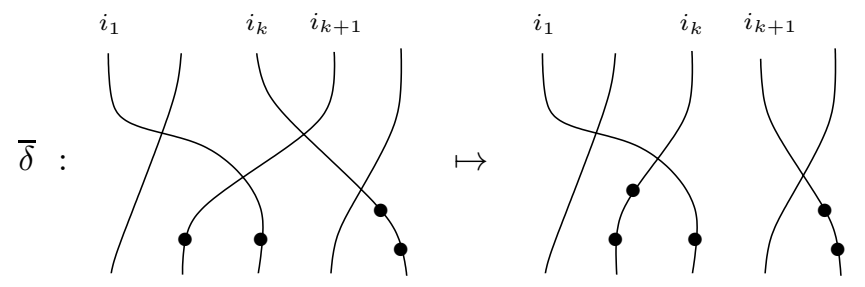

We can write $y^{\prime}={\widehat{s_{k}}}_{i} x^{u} x_{\ell, i}$ if $y=\widehat{w}_{i} x^{u}$ as above. The map $\bar{\delta}:{ }_{j^{\prime}} B_{i} \rightarrow{ }_{j} B_{i}$ is clearly injective. It is not hard to compute that $\delta_{k, j^{\prime}} y=\bar{\delta} y+$ lower-order terms:

$$
\delta_{k, j^{\prime}} y=\bar{\delta} y+\sum_{z<\bar{\delta} y} n_{z} \cdot z, \quad n_{z} \in \mathbb{Z}, \quad z \in{ }_{j} B_{i},
$$

for any $y \in{ }_{j^{\prime}} B_{i}$, and by lower-order terms we mean a linear combination of elements of ${ }_{j} B_{i}$ less than $\bar{\delta} y$ with respect to the order of ${ }_{j} B_{i}$.

The induction step follows, since ${ }_{j} B_{i}$ is a linearly independent set by induction hypothesis. This completes the proof of Theorem 2.5 .

The representation $\mathcal{P}_{o} \ell_{\nu}$ has a grading. Choose any $i \in \operatorname{Seq}(\nu)$ and place the unit element $1 \in \mathcal{P}_{o} \ell_{i}$ in degree 0 . This uniquely determines a grading on $\mathcal{P}_{o} \ell_{\nu}$ making it a graded module over the graded ring $R(\nu)$.

Corollary 2.6. $\mathcal{P} o \ell_{\nu}$ is a faithful graded module over the graded ring $R(\nu)$.

2.4. Properties of $R(\nu)$. From Theorem 2.5 we deduce several properties of $R(\nu)$. For each $\boldsymbol{i} \in \operatorname{Seq}(\nu)$ the subring ${ }_{i} R(\nu)_{i}$ contains the polynomial ring $\mathcal{P} o \ell(\nu, \boldsymbol{i}) \cong$ $\mathbb{Z}\left[x_{1, i}, x_{2, i}, \ldots, x_{m, i}\right]$. We differentiate between the ring $\mathcal{P} o \ell(\nu, i)$ and the abelian group $\mathcal{P} o \ell_{i}$ on which we defined the action. The direct product

$$
\mathcal{P} o \ell(\nu)=\prod_{i \in \operatorname{Seq}(\nu)} \mathcal{P} o \ell(\nu, i)
$$

is a commutative subring of $R(\nu)$.

Proposition 2.7. $R(\nu)$ is a free $\mathcal{P} o \ell(\nu)$-module of rank $m$ ! with respect to both left and right multiplication actions of $\mathcal{P}$ ol $(\nu)$.

Proof. For each permutation $w \in S_{m}$ choose a minimal representative $\widetilde{w}$ and form

$$
\widehat{w}=\sum_{i \in \operatorname{Seq}(\nu)} \widehat{w}_{i}
$$

Theorem 2.5 implies that the set $\{\widehat{w}\}_{w \in S_{n}}$ is a basis of $R(\nu)$ as a free graded module over $\operatorname{Po} \ell(\nu)$ under the right multiplication action of the latter. The left multiplication case follows by applying the anti-involution $\psi$ of $R(\nu)$.

The symmetric group $S_{m}$ acts on $\mathcal{P} o \ell(\nu)$ by permuting strands (which carry labels and dots). Let $\operatorname{Sym}(\nu)=\operatorname{Po\ell }(\nu)^{S_{m}}$ be the subring of $S_{m}$-invariants. It is naturally isomorphic to the tensor product of rings of symmetric polynomials

$$
\operatorname{Sym}(\nu) \cong \bigotimes_{i \in \operatorname{Supp}(\nu)} \mathbb{Z}\left[x_{1}, \ldots, x_{\nu_{i}}\right]^{S_{\nu_{i}}}
$$

over vertices $i$ in $\operatorname{Supp}(\nu)$, with the number of variables $\nu_{i}$. 
Example 2.8. The following elements are generators of $\operatorname{Sym}(2 i+j)$ :

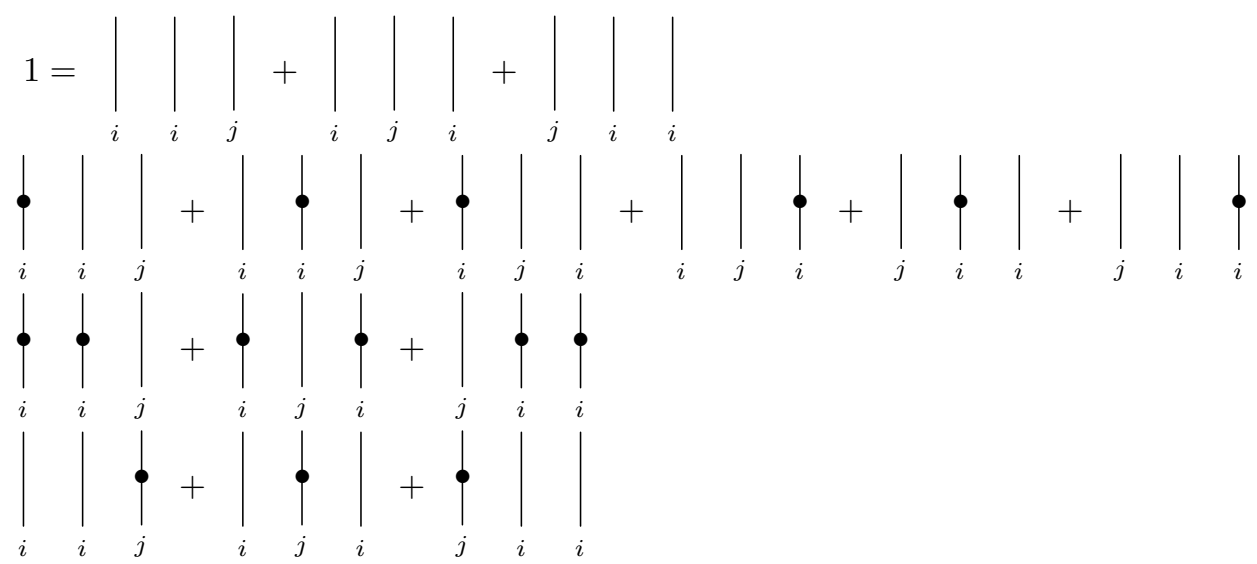

Consider the inclusions of rings

$$
\operatorname{Sym}(\nu) \subset \mathcal{P} o \ell(\nu) \subset R(\nu) .
$$

Each subsequent ring is a free rank $m$ ! module over the previous ring. Therefore, $R(\nu)$ is a free module of rank $(m !)^{2}$ over $\operatorname{Sym}(\nu)$. Moreover, a simple computation shows that $\operatorname{Sym}(\nu)$ belongs to the center of $R(\nu)$. The converse is true as well.

Theorem 2.9. $\operatorname{Sym}(\nu)$ is the center of $R(\nu)$.

Proof. The multiplication map

$$
\begin{aligned}
{ }_{i} R(\nu)_{k} & \longrightarrow{ }_{j} R(\nu)_{k} \\
y & \mapsto{ }_{j} 1_{i} y
\end{aligned}
$$

by ${ }_{j} 1_{i}$ is injective, since the composition ${ }_{i} 1_{j j} 1_{i}$ is injective, being a certain product of sums of $x_{a, i}$ 's (use Theorem 2.5) ).

A central element $z \in Z(R(\nu))$ decomposes

$$
z=\sum_{i \in \operatorname{Seq}(\nu)} z_{i}, \quad z_{i}=1_{i} z=z 1_{i} .
$$

In particular, $z_{i}$ is a central element of ${ }_{i} R(\nu)_{i}$. Let $j=j_{1}^{\nu_{1}} j_{2}^{\nu_{2}} \ldots j_{r}^{\nu_{r}}$, for some order $j_{1} \ldots j_{r}$ of vertices that appear in $\nu$. The ring ${ }_{j} R(\nu)_{j}$ is the tensor product of nil-Hecke rings

$$
{ }_{j} R(\nu)_{j} \cong \bigotimes_{t=1}^{r} N H_{\nu_{t}}
$$

and its center is isomorphic to the tensor product of centers of nil-Hecke rings, which are known to be symmetric polynomials in $\nu_{t}$ variables. Moreover, the composition

$$
\begin{array}{r}
\operatorname{Sym}(\nu) \longrightarrow Z(R(\nu)) \longrightarrow Z\left({ }_{j} R(\nu)_{j}\right) \\
z \longmapsto z_{j}
\end{array}
$$

is an isomorphism.

Subtracting an element of $\operatorname{Sym}(\nu)$, we can assume that a central element $z$ has $z_{j}=0$. Since for all $\boldsymbol{i}$,

$$
0=z_{j}\left({ }_{j} 1_{i}\right)=z\left({ }_{j} 1_{i}\right)=\left({ }_{j} 1_{i}\right) z=\left({ }_{j} 1_{i}\right) z_{i},
$$


we get $z_{i}=0$ since the multiplication by ${ }_{j} 1_{i}$ is injective.

\section{Corollary 2.10.}

1) $R(\nu)$ is a free rank $(m !)^{2}$ module over its center $\operatorname{Sym}(\nu)$.

2) $R(\nu)$ is free as a graded module over $\operatorname{Sym}(\nu)$.

The ring $\operatorname{Sym}(\nu)$ is $\mathbb{Z}_{+}$-graded. Any finitely-generated free graded $\operatorname{Sym}(\nu)$ module has a graded rank invariant which lies in $\mathbb{N}\left[q, q^{-1}\right]$. The graded rank of the module $\operatorname{Sym}(\nu)\{a\}$ whose grading starts in degree $a$ is $q^{a}$, and the graded rank is the additive under the direct sum. It is not hard to write a combinatorial formula for the graded rank of $R(\nu)$; we leave it to the reader as an exercise.

\section{Corollary 2.11.}

1) $R(\nu)$ is both left and right Noetherian.

2) $R(\nu)$ is indecomposable.

Indecomposability is equivalent to 1 being the only central idempotent in the ring. Note that $R(\nu)$ is "almost" positively graded. Precisely, it is zero in degrees less that $-\sum_{i} \nu_{i}\left(\nu_{i}-1\right)$.

2.5. Representations. In this and the following sections we assume that $R(\nu)$ is defined over a field $\mathbb{k}$ rather than over $\mathbb{Z}$. All earlier results of $R(\nu)$ remain valid over $\mathbb{k}$. We view $R(\nu)$ as a graded $\mathbb{k}$-algebra with every element of $\mathbb{k}$ in degree 0 . Let $R(\nu)-\bmod$ be the category of finitely-generated graded left $R(\nu)$-modules, let $R(\nu)$-fmod be the category of finite-dimensional graded $R(\nu)$-modules, and let $R(\nu)$ - pmod be the category of projective objects in $R(\nu)-\bmod$. The morphisms in each of these three categories are grading-preserving module homomorphisms. The first two categories are abelian. We have a diagram of categories and inclusions:

$$
R(\nu)-\text { fmod } \subset R(\nu)-\bmod \supset R(\nu)-\text { pmod. }
$$

From now on, by an $R(\nu)$-module we mean a left graded finitely-generated $R(\nu)$ module, unless otherwise specified. For any two $R(\nu)$-modules $M, N$ denote by $\operatorname{Hom}(M, N)$ or $\operatorname{Hom}_{R(\nu)}(M, N)$ the $\mathbb{k}$-vector space of grading-preserving homomorphisms, and by

$$
\operatorname{HOM}(M, N):=\bigoplus_{a \in \mathbb{Z}} \operatorname{Hom}(M, N\{a\}),
$$

the $\mathbb{Z}$-graded $\mathbb{k}$-vector space of all $R(\nu)$-module morphisms. Here $N\{a\}$ denotes $N$ with the grading shifted up by $a$. By a simple $R(\nu)$-module we mean a simple object in the category $R(\nu)$-mod. We denote by $\mathrm{Sym}^{+}(\nu)$ the unique graded maximal central ideal of $\operatorname{Sym}(\nu)$. It is spanned by $S_{m}$-invariant polynomials without the constant term.

Proposition 2.12. A simple $R(\nu)$-module $S$ is finite-dimensional and $\mathrm{Sym}^{+}(\nu)$ acts by 0 on it. $\operatorname{Hom}(S, S\{a\})=0$ if $a \neq 0$, and $S$ remains simple when viewed as an $S$-module without the grading.

Proof. The first part and the first claim in the second part of the proposition are obvious. The reference for the last statement is Theorem 4.4.4(v) in [38; see also Theorems 4.4.6 and 9.6.8 in 38. 
Hence, $S$ is a (graded) module over the finite-dimensional quotient algebra

$$
R^{\prime}(\nu)=R(\nu) / \operatorname{Sym}^{+}(\nu) R(\nu) .
$$

Note that $\operatorname{dim}_{\mathbb{k}} R^{\prime}(\nu)=(m !)^{2}$, and, up to isomorphism and grading shifts, there are only finitely many simple $R(\nu)$-modules. We choose one representative $S_{b}$ from each equivalence class, denote the set of equivalence classes by $\mathbf{B}_{\nu}^{\prime}$, and define

$$
\mathbf{B}^{\prime} \stackrel{\text { def }}{=} \bigsqcup_{\nu \in \mathbb{N}[I]} \mathbf{B}_{\nu}^{\prime}
$$

We expect a bijection between $\mathbf{B}^{\prime}$ and the Lusztig-Kashiwara canonical basis $\mathbf{B}$, hence we use a similar notation. Thus, any simple $R(\nu)$-module is isomorphic to $S_{b}\{a\}$ for a unique $b \in \mathbf{B}_{\nu}^{\prime}$ and $a \in \mathbb{Z}$ (recall that we are considering only graded modules). We do not specify the grading shift for $S_{b}$ yet (but see the end of Section 3.2.

Each module in $R(\nu)$-fmod has finite length composition series with subsequent quotients - simple modules. The Grothendieck group $G_{0}(R(\nu))$ of $R(\nu)-\mathrm{fmod}$ is a free $\mathbb{Z}\left[q, q^{-1}\right]$-module with the basis $\left\{\left[S_{b}\right]\right\}_{b \in \mathbf{B}_{\nu}^{\prime}}$ and the multiplication by $q$ corresponding to the grading shift up by 1 .

The abelian category $R(\nu)$ - mod has the Krull-Schmidt unique direct sum decomposition property for modules. Objects $P_{\boldsymbol{i}}, \boldsymbol{i} \in \operatorname{Seq}(\nu)$, belong to its subcategory $R(\nu)$-pmod of projective modules.

Each simple $S_{b}$ has a unique (up to isomorphism) indecomposable projective cover, denoted $P_{b}$. We have $\operatorname{HOM}\left(P_{b}, S_{b}\right) \cong \operatorname{Hom}\left(P_{b}, S_{b}\right) \cong \operatorname{End}\left(S_{b}\right)$. An indecomposable object of $R(\nu)$-pmod is isomorphic to $P_{b}\{a\}$ for a unique $b \in \mathbf{B}_{\nu}^{\prime}$ and $a \in \mathbb{Z}$. Any object of $R(\nu)$-pmod has a unique, up to isomorphism, direct sum decomposition into indecomposables. The Grothendieck group of $R(\nu)$-pmod is a free $\mathbb{Z}\left[q, q^{-1}\right]$-module with the basis $\left\{\left[P_{b}\right]\right\}_{b \in \mathbf{B}_{\nu}^{\prime}}$ given by the images $\left[P_{b}\right]$ of indecomposable projectives. Denote this Grothendieck group by $K_{0}(R(\nu))$.

Recall that for a right, respectively left, $R(\nu)$-module $M$ we denote by $M^{\psi}$ the left, respectively right, $R(\nu)$-module $M$ with the action twisted by $\psi$. For $P \in R(\nu)$-pmod, let $\bar{P}=\operatorname{HOM}(P, R(\nu))^{\psi}$. This is a graded projective left $R(\nu)$ module and ${ }^{-}$is a contravariant self-equivalence in $R(\nu)$-pmod. We have $\overline{P_{i}} \cong P_{i}$ for each $i \in \operatorname{Seq}(\nu)$, and, more generally, $\overline{P_{i}\{a\}} \cong P_{i}\{-a\}$. This self-equivalence induces a $\mathbb{Z}\left[q, q^{-1}\right]$-antilinear involution on $K_{0}(R(\nu))$, also denoted ${ }^{-}$.

There is a $\mathbb{Z}\left[q, q^{-1}\right]$-bilinear pairing

$$
\begin{aligned}
& (,): K_{0}(R(\nu)) \times G_{0}(R(\nu)) \longrightarrow \mathbb{Z}\left[q, q^{-1}\right], \\
& ([P],[M]):=\operatorname{gdim}_{\mathbb{k}}\left(P^{\psi} \otimes_{R(\nu)} M\right) .
\end{aligned}
$$

When the field $\mathbb{k}$ is algebraically closed, $\operatorname{End}\left(S_{b}\right) \cong \mathbb{k}$, and the bases $\left\{\left[P_{b}\right]\right\}_{b}$ and $\left\{\left[S_{b}\right]\right\}_{b}$ are dual, possibly up to rescaling by powers of $q$ and permutation of elements. In this case $G_{0}(R(\nu))$ and $K_{0}(R(\nu))$ are dual free $\mathbb{Z}\left[q, q^{-1}\right]$-modules. We will show in Section 3.2 that, over any field $\mathbb{k}$, simples $S_{b}$ are absolutely irreducible and the above pairing is perfect without any restrictions on $\mathbb{k}$.

There is a $\mathbb{Z}\left[q, q^{-1}\right]$-bilinear form, also denoted $($,$) ,$

$$
(,): K_{0}(R(\nu)) \times K_{0}(R(\nu)) \longrightarrow \mathbb{Z}\left[q^{-1}, q\right] \cdot(\nu)_{q},
$$


where

$$
(\nu)_{q}=\operatorname{gdim}(\operatorname{Sym}(\nu))=\prod_{i \in \Gamma}\left(\prod_{a=1}^{\nu_{i}} \frac{1}{1-q^{2 a}}\right)
$$

and

$$
([P],[Q])=\operatorname{gdim}_{\mathbb{k}}\left(P^{\psi} \otimes_{R(\nu)} Q\right) .
$$

Since $P^{\psi} \otimes_{R(\nu)} Q \cong Q^{\psi} \otimes_{R(\nu)} P$, the form is symmetric. It follows from Theorem 2.5 that ${ }_{i} R(\nu)_{j} \cong{ }_{i} P \otimes_{R(\nu)} P_{j}$ is a free graded $\operatorname{Sym}(\nu)$-module for any $\boldsymbol{i}, \boldsymbol{j}$. Therefore, $P^{\psi} \otimes_{R(\nu)} Q$ is a free graded $\operatorname{Sym}(\nu)$-module of finite rank for any $P, Q$ as above, and the form takes values in $\mathbb{Z}\left[q^{-1}, q\right] \cdot(\nu)_{q}$. We have

$$
\left(\left[P_{j}\right],\left[P_{i}\right]\right)=\operatorname{gdim}\left({ }_{j} P \otimes_{R(\nu)} P_{i}\right)=\operatorname{gdim}\left({ }_{j} R(\nu)_{i}\right) .
$$

Define the character $\operatorname{ch}(M)$ of a graded finitely-generated $R(\nu)$-module $M$ as

$$
\operatorname{ch}(M)=\sum_{i \in \operatorname{Seq}(\nu)} \operatorname{gdim}\left(1_{i} M\right) \cdot \boldsymbol{i} .
$$

The character is an element of the free $\mathbb{Z}((q))$-module with the basis $\operatorname{Seq}(\nu)$; when $M$ is finite-dimensional, $\operatorname{ch}(M)$ is an element of the free $\mathbb{Z}\left[q, q^{-1}\right]$-module with basis $\operatorname{Seq}(\nu)$. We abbreviate $\operatorname{gdim}\left(1_{i} M\right)$ to $\operatorname{ch}(M, \boldsymbol{i})$,

$$
\operatorname{ch}(M)=\sum_{i \in \operatorname{Seq}(\nu)} \operatorname{ch}(M, \boldsymbol{i}) \cdot \boldsymbol{i} .
$$

Let $\operatorname{Seqd}(\nu)$ be the set of all expressions $i_{1}^{\left(n_{1}\right)} i_{2}^{\left(n_{2}\right)} \ldots i_{r}^{\left(n_{r}\right)}$ such that $n_{1}, \ldots, n_{r} \in$ $\mathbb{N}$ and $\sum_{a=1}^{r} n_{a} i_{a}=\nu$. For instance,

$$
\operatorname{Seqd}(2 i+j)=\left\{i i j, i j i, j i j, i^{(2)} j, j i^{(2)}\right\} .
$$

To $i \in \operatorname{Seqd}(\nu)$ we assign the idempotent

$$
1_{i}=e_{i_{1}, n_{1}} \otimes e_{i_{2}, n_{2}} \otimes \cdots \otimes e_{i_{r}, n_{r}},
$$

given by the tensor product of minimal idempotents $e_{i, n}$ in the nil-Hecke rings; see Section 2.2 ,

Let $\boldsymbol{i} !=\left[n_{1}\right]$ ! .. [n $\left.n_{r}\right]$ !, and $\widehat{\boldsymbol{i}}$ be the element of $\operatorname{Seq}(\nu)$ given by expanding $\boldsymbol{i}$,

$$
\widehat{\boldsymbol{i}}=i_{1} \ldots i_{1} i_{2} \ldots i_{2} \ldots i_{r} \ldots i_{r} .
$$

$\widehat{\boldsymbol{i}}=\boldsymbol{i}$ iff $\boldsymbol{i} \in \operatorname{Seq}(\nu)$. We have the equality of graded dimensions

$$
\operatorname{gdim}\left(1_{\widehat{\boldsymbol{i}}} M\right)=q^{-\langle i\rangle} \boldsymbol{i} ! \cdot \operatorname{gdim}\left(1_{i} M\right),\langle\boldsymbol{i}\rangle=\sum_{k=1}^{r} \frac{n_{k}\left(n_{k}-1\right)}{2},
$$

which follows from the structure of the nil-Hecke algebra. Let

$$
\operatorname{ch}(M, \boldsymbol{i})=q^{-\langle i\rangle} \cdot \operatorname{gdim}\left(1_{i} M\right)
$$

then

$$
\operatorname{ch}(M, \widehat{i})=i ! \cdot \operatorname{ch}(M, i) .
$$

In particular, $\operatorname{ch}(M)$ determines $\operatorname{ch}(M, i)$ for any $i \in \operatorname{Seqd}(\nu)$.

For $\boldsymbol{i} \in \operatorname{Seqd}(\nu)$ define the left graded projective module

$$
P_{i}=R(\nu) \psi\left(1_{i}\right)\{-\langle i\rangle\},
$$


and the right graded projective module

$$
{ }_{i} P=1_{i} R(\nu)\{-\langle i\rangle\} .
$$

We have $P_{\widehat{i}} \cong P_{i}^{i !}$ and ${ }_{i} P \cong{ }_{i} P^{i !}$. For instance,

$$
P_{i i} \cong P_{i^{(2)}}^{[2] !}=P_{i^{(2)}}^{q+q^{-1}}=P_{i^{(2)}}\{1\} \oplus P_{i^{(2)}}\{-1\} .
$$

Moreover,

$$
\operatorname{ch}(M, \boldsymbol{i})=\operatorname{gdim}\left({ }_{i} P \otimes_{R(\nu)} M\right)=\operatorname{gdim}\left(\operatorname{HOM}\left(\mathrm{P}_{i}, \mathrm{M}\right)\right) .
$$

Given two or more sequences in $\operatorname{Seqd}(\nu)$ that differ only in several neighboring terms, we denote identical parts in them via dots. For instance, ...ij ... and $\ldots j i \ldots$ denote a pair of sequences $\boldsymbol{i}^{\prime} i j \boldsymbol{i}^{\prime \prime}$ and $\boldsymbol{i}^{\prime} j i \boldsymbol{i}^{\prime \prime}$ for some sequences $\boldsymbol{i}^{\prime}, \boldsymbol{i}^{\prime \prime}$.

Proposition 2.13. There are isomorphisms of graded projective right $R(\nu)$-modules

$$
\begin{aligned}
\ldots i j \ldots P & \cong \ldots, j i \ldots P \text { if } i \cdot j=0, \\
\ldots i j i \ldots P & \cong P_{\ldots i{ }^{(2)} j \ldots} \oplus P_{\ldots j i^{(2)} \ldots} \text { if } i \cdot j=-1,
\end{aligned}
$$

and isomorphisms of graded projective left $R(\nu)$-modules

$$
\begin{aligned}
& P_{\ldots i j \ldots} \cong P_{\ldots j i \ldots,} \text { if } i \cdot j=0, \\
& P_{\ldots i j i \ldots} \cong P_{\ldots i(2)} \ldots \oplus P_{\ldots j i^{(2)} \ldots} \text { if } i \cdot j=-1 .
\end{aligned}
$$

Proof. It suffices to show the isomorphisms for right projective modules; application of the anti-involution $\psi$ would imply the corresponding isomorphisms for left projective modules. Multiplication by the diagram

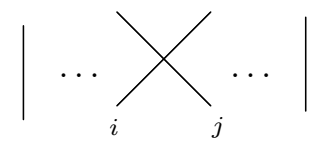

is a grading-preserving isomorphism between $\ldots i j \ldots P$ and $\ldots j i \ldots P$ if $i \cdot j=0$.

Consider grading-preserving maps

$$
\begin{array}{lll}
B_{0} & : & \ldots i j i \ldots P \longrightarrow \ldots i(2) \\
B_{1} & : & \ldots P \oplus \ldots j i^{(2)} \ldots P \\
B^{(2)} j \ldots P \oplus \ldots j i^{(2)} \ldots P \longrightarrow \ldots i j i \ldots P
\end{array}
$$

given by matrices of diagrams

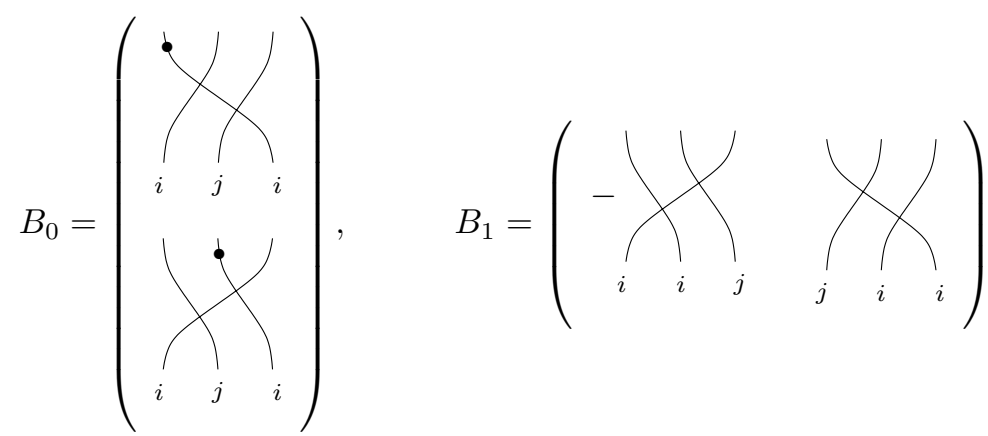

Notice that the top entry in $B_{0}$ ends with the projector $e_{2, i}$ which takes $1 \ldots i i \ldots$ to $1_{\ldots i(2) \ldots}$, and we view this entry as a homomorphism

$$
\ldots i j i \ldots P \longrightarrow \ldots i i j \ldots P \longrightarrow \ldots i(2) j \ldots P
$$


ditto for the bottom entry in $B_{0}$. The degree of each diagram in $B_{0}$ is 1 , therefore the map $B_{0}$ is grading-preserving since the grading shifts for the sequences differ by $\left\langle\ldots i^{(2)} j \ldots\right\rangle-\langle\ldots i j i \ldots\rangle=1$.

We view the first entry in $B_{1}$ as the composition

$$
\ldots i^{(2)} j \ldots P \subset \ldots i i j \ldots P \longrightarrow \ldots i j i \ldots P,
$$

and there is no need to write the corresponding idempotent (the same for the second entry). We compute

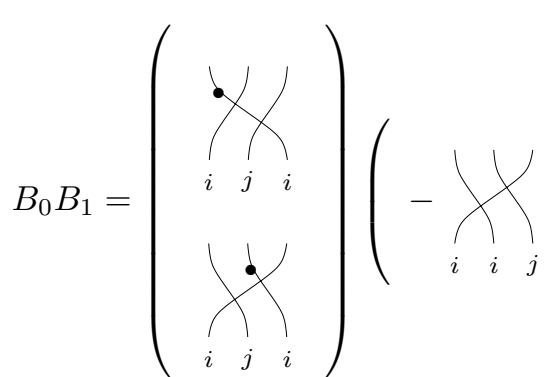

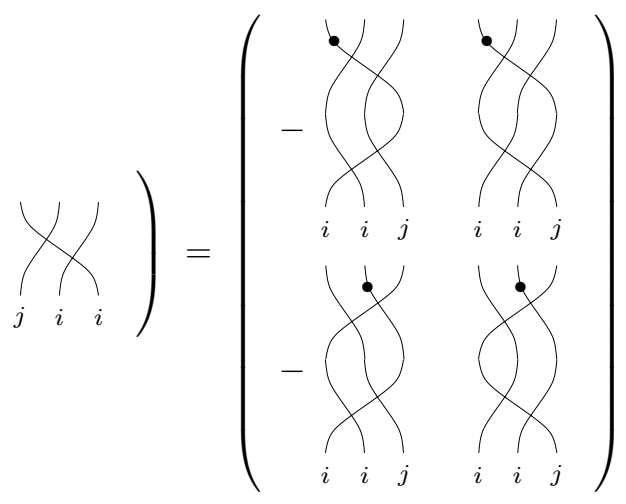

In the last matrix above the diagonal terms are idempotents $1_{i^{(2)} j}$ and $1_{j i^{(2)}}$, giving identity maps of projectives $\ldots i^{(2)} j \ldots P$ and $\ldots j i^{(2)} \ldots P$, respectively.

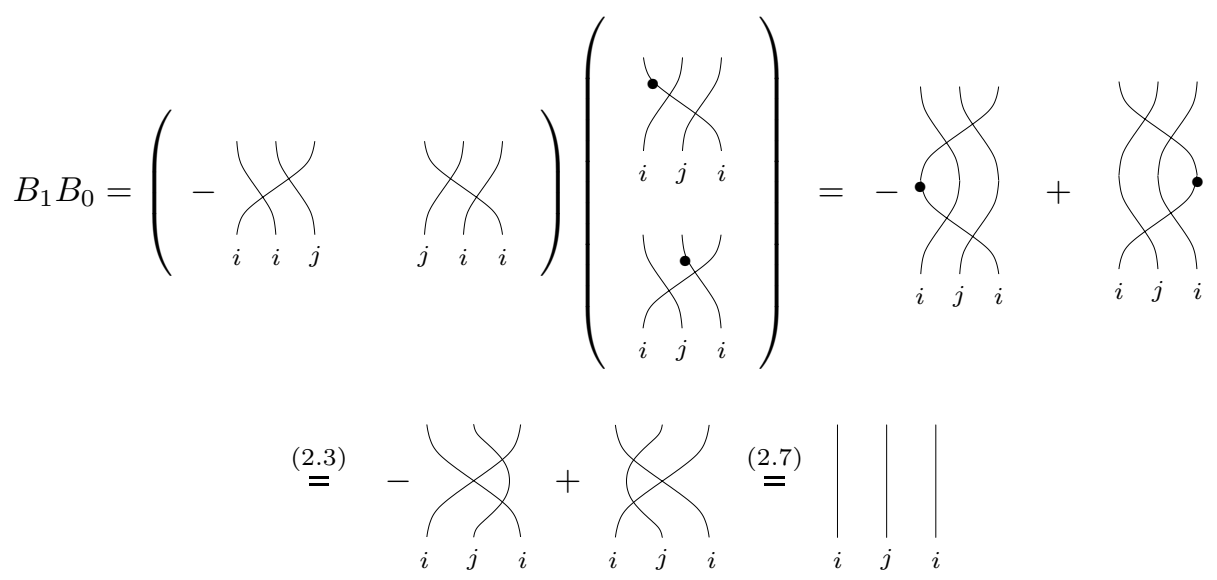

Therefore, $B_{0}, B_{1}$ are isomorphisms, and the second isomorphism follows. 
Corollary 2.14. For any $M$ in $R(\nu)$ - mod there are isomorphisms of graded vector spaces

$$
\begin{aligned}
1_{\ldots i j \ldots M} & \cong 1_{\ldots j i . . M} \text { if } i \cdot j=0, \\
\left.1_{\ldots i j i \ldots M} . \ldots 1\right\} & \cong 1_{\ldots i(2)} \ldots M \oplus 1_{\ldots j i(2)} \ldots M \text { if } i \cdot j=-1 .
\end{aligned}
$$

Corollary 2.15. The following character equalities hold for any graded finitelygenerated $R(\nu)$-module $M$,

$$
\begin{aligned}
& \operatorname{ch}(M, \ldots i j \ldots)=\operatorname{ch}(M, \ldots, j i \ldots) \text { if } i \cdot j=0 \\
& \operatorname{ch}(M, \ldots i j i \ldots)=\operatorname{ch}\left(M, \ldots i^{(2)} j \ldots\right)+\operatorname{ch}\left(M, \ldots j i^{(2)} \ldots\right) \text { if } i \cdot j=-1, \\
& \operatorname{ch}\left(M, \ldots i^{(a)} i^{(b)} \ldots\right)=\left[\begin{array}{c}
a+b \\
a
\end{array}\right] \operatorname{ch}\left(M, \ldots i^{(a+b)} \ldots\right) .
\end{aligned}
$$

2.6. Induction and restriction. Suppose we have an inclusion of rings $\iota: B \hookrightarrow A$ which is not necessarily unital: $e=\iota(1)$ is only an idempotent in $A$. The induction functor between categories of unital modules

$$
B-\bmod \stackrel{\text { Ind }}{\longrightarrow} A-\bmod , \quad M \longmapsto A \otimes_{B} M
$$

is isomorphic to the functor $M \longmapsto A e \otimes_{B} M$. Its right adjoint

$$
\text { Res : } A-\bmod \longrightarrow B-\bmod
$$

takes $M$ to $e M$, viewed as a $B$-module.

The inclusion of graded rings

$$
\iota_{\nu, \nu^{\prime}}: R(\nu) \otimes R\left(\nu^{\prime}\right) \hookrightarrow R\left(\nu+\nu^{\prime}\right)
$$

is described by putting the diagrams next to each other. It takes the idempotent $1_{i} \otimes 1_{j}$ to $1_{i j}$ and the unit element to an idempotent of $R\left(\nu+\nu^{\prime}\right)$ denoted $1_{\nu, \nu^{\prime}}$.

Proposition 2.16. $1_{\nu, \nu^{\prime}} R\left(\nu+\nu^{\prime}\right)$ is a free graded left $R(\nu) \otimes R\left(\nu^{\prime}\right)$-module.

Proof. The minimal representative $w$ of a left $S_{|\nu|} \times S_{\left|\nu^{\prime}\right|}$-coset in $S_{|\nu|+\left|\nu^{\prime}\right|}$ gives rise to the diagram

$$
{ }_{i j} \widehat{w} \in{ }_{i j} R\left(\nu+\nu^{\prime}\right)_{w^{-1}(i j)}
$$

of the minimal presentation of $w$ with top ends of strands labelled by the sequence $\boldsymbol{i} \boldsymbol{j}$ for $\boldsymbol{i} \in \operatorname{Seq}(\nu)$ and $\boldsymbol{j} \in \operatorname{Seq}\left(\nu^{\prime}\right)$ :

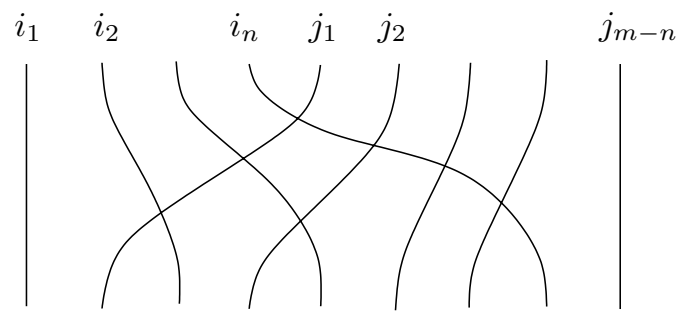

The set of elements

$$
\widehat{w}=\sum_{i \in \nu, j \in \nu^{\prime}} i j \widehat{w}
$$

over all cosets, is a basis of $1_{\nu, \nu^{\prime}} R\left(\nu+\nu^{\prime}\right)$ as a free graded left $R(\nu) \otimes R\left(\nu^{\prime}\right)$ module. 
We denote the restriction and induction functors for the inclusion

$$
R(\nu) \otimes R\left(\nu^{\prime}\right) \subset R\left(\nu+\nu^{\prime}\right)
$$

by $\operatorname{Res}_{\nu, \nu^{\prime}}$ and $\operatorname{Ind}_{\nu, \nu^{\prime}}$, respectively.

Corollary 2.17. The restriction functor $\operatorname{Res}_{\nu, \nu^{\prime}}$ takes projectives to projectives.

Given a quadruple $\left(\nu, \nu^{\prime}, \nu^{\prime \prime}, \nu^{\prime \prime \prime}\right)$ with $\nu+\nu^{\prime}=\nu^{\prime \prime}+\nu^{\prime \prime \prime}$, let

$$
{ }_{\nu, \nu^{\prime}} R_{\nu^{\prime \prime}, \nu^{\prime \prime \prime}}=1_{\nu} \otimes 1_{\nu^{\prime}} R\left(\nu+\nu^{\prime}\right) 1_{\nu^{\prime \prime}} \otimes 1_{\nu^{\prime \prime \prime}} .
$$

Proposition 2.18. Graded $\left(R(\nu) \otimes R\left(\nu^{\prime}\right), R\left(\nu^{\prime \prime}\right) \otimes R\left(\nu^{\prime \prime \prime}\right)\right)$-bimodule ${ }_{\nu, \nu^{\prime}} R_{\nu^{\prime \prime}, \nu^{\prime \prime \prime}}$ has a filtration by graded bimodules isomorphic to

$\left({ }_{\nu} R_{\nu-\lambda, \lambda} \otimes_{\nu^{\prime}} R_{\nu^{\prime}+\lambda-\nu^{\prime \prime \prime}, \nu^{\prime \prime \prime}-\lambda}\right) \otimes_{R^{\prime}}\left(\nu-\lambda, \nu^{\prime \prime}+\lambda-\nu R_{\nu^{\prime \prime}} \otimes_{\lambda, \nu^{\prime \prime \prime}-\lambda} R_{\nu^{\prime \prime \prime}}\right)\left\{-\lambda \cdot\left(\nu^{\prime}+\lambda-\nu^{\prime \prime \prime}\right)\right\}$,

where $R^{\prime}=R(\nu-\lambda) \otimes R(\lambda) \otimes R\left(\nu^{\prime}+\lambda-\nu^{\prime \prime \prime}\right) \otimes R\left(\nu^{\prime \prime \prime}-\lambda\right)$, over all $\lambda \in \mathbb{N}[I]$ such that every term above is in $\mathbb{N}[I]$.

Proof. This proposition is a version of the Mackey's induction-restriction theorem for inclusion of maximal parabolic subgroups $S_{m-n} \times S_{n} \subset S_{m}$. The statement and its proof are best illustrated by the diagram

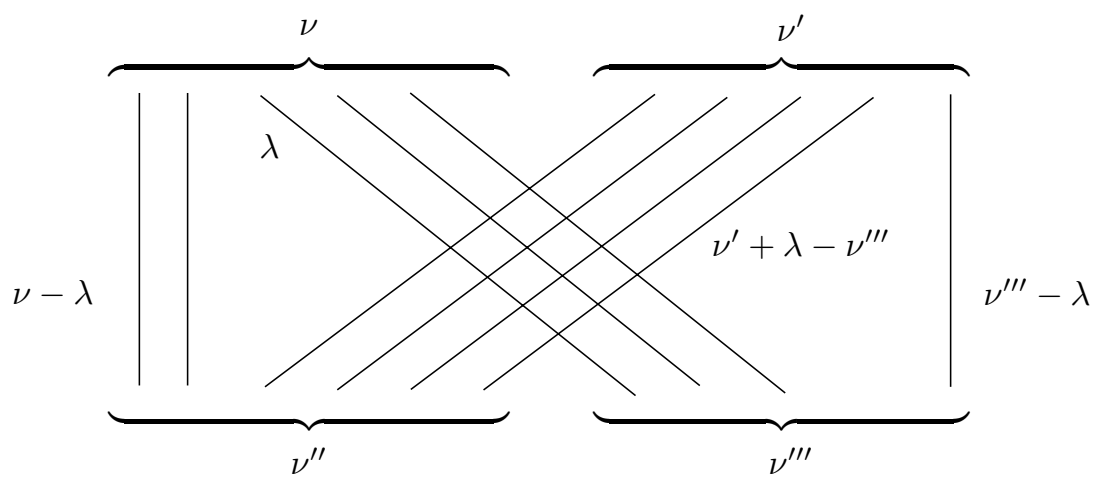

These diagrams, over all $\lambda$ (summing over all colorings of strands) will provide generators for the subquotient bimodules that appear in the proposition. The grading shift $-\lambda \cdot\left(\nu^{\prime}+\lambda-\nu^{\prime \prime \prime}\right)$ is the degree of the intersection diagram of $|\lambda|$ parallel lines colored by any $i \in \operatorname{Seq}(\lambda)$ and $\left|\nu^{\prime}+\lambda-\nu^{\prime \prime \prime}\right|$ parallel lines colored by any $\boldsymbol{j} \in \operatorname{Seq}\left(\nu^{\prime}+\lambda-\nu^{\prime \prime \prime}\right)$.

We have

$$
\operatorname{Ind}_{\nu, \nu^{\prime}}\left(P_{i} \otimes P_{j}\right) \cong P_{i j}
$$

for $\boldsymbol{i} \in \operatorname{Seq}(\nu), \boldsymbol{j} \in \operatorname{Seq}\left(\nu^{\prime}\right)$. By passing to direct summands, we see that the formula holds more generally, for $\boldsymbol{i} \in \operatorname{Seqd}(\nu), \boldsymbol{j} \in \operatorname{Seqd}\left(\nu^{\prime}\right)$.

A shuffle $\boldsymbol{k}$ of a pair of sequences $\boldsymbol{i} \in \operatorname{Seq}(\nu), \boldsymbol{j} \in \operatorname{Seq}\left(\nu^{\prime}\right)$ is a sequence together with a choice of subsequence isomorphic to $\boldsymbol{i}$ such that $\boldsymbol{j}$ is the complementary subsequence. Shuffles of $\boldsymbol{i}, \boldsymbol{j}$ are in a bijection with the minimal coset representatives of $S_{|\nu|} \times S_{\left|\nu^{\prime}\right|}$ in $S_{|\nu|+\left|\nu^{\prime}\right|}$. We denote by $\operatorname{deg}(\boldsymbol{i}, \boldsymbol{j}, \boldsymbol{k})$ the degree of the diagram in 
$R\left(\nu+\nu^{\prime}\right)$ naturally associated to the shuffle; see an example below:

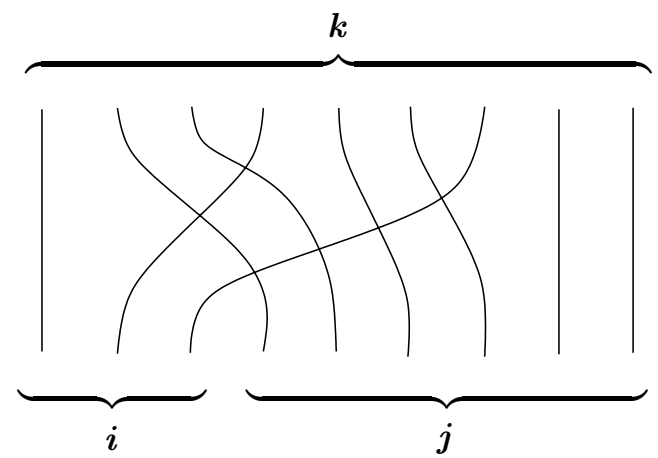

When the meaning is clear, we will also denote by $\boldsymbol{k}$ the underlying sequence of the shuffle $\boldsymbol{k}$.

Proposition 2.19. For any $\boldsymbol{k} \in \operatorname{Seq}\left(\nu+\nu^{\prime}\right)$,

$$
\begin{aligned}
\operatorname{Res}_{\nu, \nu^{\prime}} P_{k} & \cong \bigoplus_{\boldsymbol{i}, \boldsymbol{j}} P_{i} \otimes P_{j}\{\operatorname{deg}(\boldsymbol{i}, \boldsymbol{j}, \boldsymbol{k})\} \\
\operatorname{Res}_{\nu, \nu^{\prime}}\left({ }_{k} P\right) & \cong \bigoplus_{i * j=k} P \otimes_{j} P\{\operatorname{deg}(\boldsymbol{i}, \boldsymbol{j}, \boldsymbol{k})\},
\end{aligned}
$$

the sum over all ways to represent $\boldsymbol{k}$ as a shuffle of $\boldsymbol{i} \in \operatorname{Seq}(\nu)$ and $\boldsymbol{j} \in \operatorname{Seq}\left(\nu^{\prime}\right)$.

The proposition follows immediately from the structure of bimodules ${ }_{\nu, \nu^{\prime}} R_{\nu+\nu^{\prime}}$ and ${ }_{\nu+\nu^{\prime}} R_{\nu, \nu^{\prime}}$.

Given two functions $f$ and $g$ on sets $\operatorname{Seq}(\nu)$ and $\operatorname{Seq}\left(\nu^{\prime}\right)$, respectively, with values in some commutative ring which contains $\mathbb{Z}\left[q, q^{-1}\right]$, we define their (quantum) shuffle product $f \omega g$ (see [30] and references therein) as a function on $\operatorname{Seq}\left(\nu+\nu^{\prime}\right)$ given by

$$
(f w g)(\boldsymbol{k})=\sum_{i, \boldsymbol{j}} q^{\operatorname{deg}(\boldsymbol{i}, \boldsymbol{j}, \boldsymbol{k})} f(\boldsymbol{i}) g(\boldsymbol{j}),
$$

the sum is over all ways to represent $\boldsymbol{k}$ as a shuffle of $\boldsymbol{i}$ and $\boldsymbol{j}$.

Lemma 2.20. For $M \in R(\nu)-\bmod$ and $N \in R\left(\nu^{\prime}\right)-\bmod$ we have

$$
\operatorname{ch}\left(\operatorname{Ind}_{\nu, \nu^{\prime}}(M \otimes N)\right)=\operatorname{ch}(M) \omega \operatorname{ch}(N) .
$$

Proof. This lemma follows at once from the last proposition and formula (2.48).

3. Quantum groups and the Grothendieck Ring of $R$

3.1. Homomorphism $\gamma$ of twisted bialgebras. For a graph $\Gamma$, we form the direct sum

$$
R=\bigoplus_{\nu \in \mathbb{N}[I]} R(\nu)
$$


This is a nonunital ring. By various categories of $R$-modules we will mean direct sums of corresponding categories of $R(\nu)$-modules:

$$
\begin{aligned}
& R-\bmod \stackrel{\text { def }}{=} \bigoplus_{\nu \in \mathbb{N}[I]} R(\nu)-\bmod , \\
& R-\text { fmod } \stackrel{\text { def }}{=} \bigoplus_{\nu \in \mathbb{N}[I]} R(\nu)-\mathrm{fmod}, \\
& R-\text { pmod } \stackrel{\text { def }}{=} \bigoplus_{\nu \in \mathbb{N}[I]} R(\nu)-\text { pmod. }
\end{aligned}
$$

The Grothendieck groups

$$
K_{0}(R)=\bigoplus_{\nu \in \mathbb{N}[I]} K_{0}(R(\nu)), \quad G_{0}(R)=\bigoplus_{\nu \in \mathbb{N}[I]} G_{0}(R(\nu))
$$

are the direct sums of Grothendieck groups of rings $R(\nu)$. We extend the pairings (2.43) and (2.45) to $K_{0}(R)$ and $G_{0}(R)$ by requiring that subspaces corresponding to different $\nu$ 's be orthogonal. Induction and restriction functors for the inclusion $R(\nu) \otimes R\left(\nu^{\prime}\right) \subset R\left(\nu+\nu^{\prime}\right)$, summed over all $\nu, \nu^{\prime}$, give functors

$$
\text { Ind }: R \otimes R-\bmod \longrightarrow R-\bmod , \quad \operatorname{Res}: R-\bmod \longrightarrow R \otimes R-\bmod
$$

where by $R \otimes R-\bmod$ we mean the direct sum of categories $R(\nu) \otimes R\left(\nu^{\prime}\right)-\bmod$, over all $\nu, \nu^{\prime}$. These functors restrict to subcategories of finite-dimensional modules and projective modules. Indeed, induction takes projectives to projectives. Restriction, in the case of these inclusions, also takes projectives to projectives, by Proposition 2.19 and the Krull-Shmidt property.

Thus, these functors induce maps [Ind], [Res] on Grothendieck groups $K_{0}(R)$ and $G_{0}(R)$. Note that [Res] is the sum of maps

$$
K_{0}\left(R\left(\nu+\nu^{\prime}\right)\right) \longrightarrow K_{0}(R(\nu)) \otimes K_{0}\left(R\left(\nu^{\prime}\right)\right),
$$

or

$$
G_{0}\left(R\left(\nu+\nu^{\prime}\right)\right) \longrightarrow G_{0}(R(\nu)) \otimes G_{0}\left(R\left(\nu^{\prime}\right)\right),
$$

over all $\nu, \nu^{\prime}$; the tensor products here and further are over $\mathbb{Z}\left[q, q^{-1}\right]$.

Proposition 3.1. [Ind] turns $K_{0}(R)$ and $G_{0}(R)$ into associative unital $\mathbb{Z}\left[q, q^{-1}\right]$ algebras. [Res] turns $K_{0}(R)$ and $G_{0}(R)$ into coassociative counital coalgebras over $\mathbb{Z}\left[q, q^{-1}\right]$.

Proof follows from the associativity of induction and restriction. The unit element is given by inducing with the one-dimensional module over $R(\emptyset)$. The counit is given by restricting to $R(\emptyset)$ and taking the graded dimension.

Denote the product [Ind] $\left(x_{1}, x_{2}\right)$ for $x_{1}, x_{2} \in K_{0}(R)$ simply by $x_{1} x_{2}$.

We equip $K_{0}(R) \otimes K_{0}(R)$ with the algebra structure via

$$
\left(x_{1} \otimes x_{2}\right)\left(x_{1}^{\prime} \otimes x_{2}^{\prime}\right)=q^{-\left|x_{2}\right| \cdot\left|x_{1}^{\prime}\right|} x_{1} x_{1}^{\prime} \otimes x_{2} x_{2}^{\prime}
$$

for homogeneous $x_{1}, x_{2}, x_{1}^{\prime}, x_{2}^{\prime}$, where $\left|x_{2}\right| \in \mathbb{N}[I]$ is the weight of $x_{2}$, etc.

Proposition 3.2. [Res] is an algebra homomorphism from $K_{0}(R)$ to $K_{0}(R) \otimes$ $K_{0}(R)$ with the above algebra structure.

Proof. This follows from Proposition 2.18 , 
Recall the symmetric bilinear pairing (2.45) on $K_{0}(R)$ taking values in $\mathbb{Z}\left[q, q^{-1}\right]$. $(\nu)_{q}$.

Proposition 3.3. The pairing (, ) has the following properties:

(1) $(1,1)=1$,

(2) $\left(\left[P_{i}\right],\left[P_{j}\right]\right)=\delta_{i, j}\left(1-q^{2}\right)^{-1}$ for $i, j \in I$,

(3) $\left(x, y y^{\prime}\right)=\left([\operatorname{Res}](x), y \otimes y^{\prime}\right)$, for $x, y, y^{\prime} \in K_{0}(R)$,

(4) $\left(x x^{\prime}, y\right)=\left(x \otimes x^{\prime},[\operatorname{Res}](y)\right)$, for $x, x^{\prime}, y \in K_{0}(R)$.

Proof. Since $1=\left[P_{\emptyset}\right]$, where $\emptyset$ is the empty sequence, and $P_{\emptyset}=\mathbb{k}$ as a module over $R(\emptyset)=\mathbb{k}$, the first statement follows. When $i \neq j$, vectors $\left[P_{i}\right]$ and $\left[P_{j}\right]$ lie in mutually orthogonal subspaces $K_{0}(R(i))$ and $K_{0}(R(j))$, so that $\left(\left[P_{i}\right],\left[P_{j}\right]\right)=0$. Also,

$$
\left(\left[P_{i}\right],\left[P_{i}\right]\right)=\operatorname{gdim}\left({ }_{i} R(i)_{i}\right)=\operatorname{gdim}(\mathbb{k}[x])=\left(1-q^{2}\right)^{-1} .
$$

Let $X \in R\left(\nu+\nu^{\prime}\right)$-pmod, $Y \in R(\nu)$-pmod, and $Y^{\prime} \in R\left(\nu^{\prime}\right)$-pmod. Then

$$
\begin{aligned}
& \left([X],[Y]\left[Y^{\prime}\right]\right)=\left([X],\left[\operatorname{Ind}_{\nu, \nu^{\prime}} Y \otimes Y^{\prime}\right]\right) \\
& =\operatorname{gdim}\left(X^{\psi} \otimes_{R\left(\nu+\nu^{\prime}\right)}\left(\nu+\nu^{\prime} R_{\nu, \nu^{\prime}}\right) \otimes_{R(\nu) \otimes R\left(\nu^{\prime}\right)} Y \otimes Y^{\prime}\right) \\
& =\operatorname{gdim}\left(X^{\psi}\left(1_{\nu} \otimes 1_{\nu^{\prime}}\right) \otimes_{R(\nu) \otimes R\left(\nu^{\prime}\right)} Y \otimes Y^{\prime}\right)=\left(\left[\operatorname{Res}_{\nu, \nu^{\prime}} X\right],[Y] \otimes\left[Y^{\prime}\right]\right),
\end{aligned}
$$

and statement (3) follows. A similar computation establishes (4).

We next recall $\mathbb{Q}(q)$-algebras 'f and f from [34, Section 1] and ${ }_{\mathcal{A}} \mathbf{f}$, the integral form of $\mathbf{f}$ (our $q$ is Lusztig's $v^{-1}$ ). Algebra ' $\mathbf{f}$ is a free associative $\mathbb{N}[I]$-graded algebra on generators $\theta_{i}$. The degree of $\theta_{i}$ is $i$. The tensor product ' $\mathbf{f} \otimes^{\prime} \mathbf{f}$ is equipped with an algebra structure using the rule (3.1), and with a coalgebra structure $r$ : 'f $\longrightarrow$ ' $\mathbf{f} \otimes^{\prime} \mathbf{f}$, determined by the conditions $r\left(\theta_{i}\right)=\theta_{i} \otimes 1+1 \otimes \theta_{i}$ and $r$ being an algebra homomorphism.

'f comes equipped with a bilinear form $($,$) uniquely determined by the same$ conditions as the ones in Proposition 3.3. with comultiplication $r$ taking the place of comultiplication [Res] in $K_{0}(R)$ and $\theta_{i}, \theta_{j}$ taking the place of $\left[P_{i}\right],\left[P_{j}\right]$. It has the weight space decomposition

$$
' \mathbf{f}=\bigoplus_{\nu \in \mathbb{N}[I]} \mathbf{f}_{\nu} .
$$

Let $\mathcal{I}$ be the radical of the bilinear form $($,$) . It is a two-sided ideal of ' \mathbf{f}$ and one forms the quotient algebra $\mathbf{f}=' \mathbf{f} / \mathcal{I}$, which also has the weight decomposition

$$
\mathbf{f}=\bigoplus_{\nu \in \mathbb{N}[I]} \mathbf{f}_{\nu}
$$

The bilinear form and the comultiplication $r$ descend to the quotient algebra. ' $\mathbf{f}$ and $\mathbf{f}$ come with a $\mathbb{Q}(q)$-antilinear involution ${ }^{-}$that takes $q^{n}$ to $q^{-n}$ and $\theta_{i}$ to $\theta_{i}$.

It is not hard to check that the elements

$$
\theta_{i} \theta_{j}-\theta_{i} \theta_{j} \text { for } i \cdot j=0
$$

and

$$
\left(q+q^{-1}\right) \theta_{i} \theta_{j} \theta_{i}-\theta_{i}^{2} \theta_{j}-\theta_{j} \theta_{i}^{2} \text { for } i \cdot j=-1
$$

belong to the ideal $\mathcal{I}$. The quantum version of the Gabber-Kac theorem says that $\mathcal{I}$ is generated by these elements over all pairs of vertices $i \neq j$ of the graph $\Gamma$ (for instance, see Theorem 33.1.3 in 34]). 
Define ${ }_{\mathcal{A}} \mathbf{f}$ as the $\mathbb{Z}\left[q, q^{-1}\right]$-subalgebra generated by the divided powers $\theta_{i}^{(a)}, i \in I$, $a \in \mathbb{N}$.

Proposition 3.4. There is an injective homomorphism of $\mathbb{Z}\left[q, q^{-1}\right]$-algebras $\gamma$ : ${ }_{\mathcal{A}} \mathbf{f} \longrightarrow K_{0}(R)$ that takes $\theta_{i_{1}}^{\left(a_{1}\right)} \ldots \theta_{i_{k}}^{\left(a_{k}\right)}$ to $\left[P_{i}\right]$, where $\boldsymbol{i}=i_{1}^{\left(a_{1}\right)} \ldots i_{k}^{\left(a_{k}\right)}$. This homomorphism converts the comultiplication $r$ of ${ }_{\mathcal{A}} \mathbf{f}$ into the comultiplication [Res] in $K_{0}(R)$. It takes the bilinear form on ${ }_{\mathcal{A}} \mathbf{f}$ to the bilinear form on $K_{0}(R)$ :

$$
(x, y)=(\gamma(x), \gamma(y))
$$

The bar-involution of ${ }_{\mathcal{A}} \mathbf{f}$ goes to the bar-involution of $K_{0}(R)$ under $\gamma$.

Proof. Start with the homomorphism of $\mathbb{Q}(q)$-algebras ' $\mathbf{f} \longrightarrow K_{0}(R)_{\mathbb{Q}(q)}$, where

$$
K_{0}(R)_{\mathbb{Q}(q)} \stackrel{\text { def }}{=} K_{0}(R) \otimes_{\mathbb{Z}\left[q, q^{-1}\right]} \mathbb{Q}(q),
$$

defined by the condition that it takes $\theta_{i}$ to $\left[P_{i}\right]$. There are equalities in $K_{0}(R)_{\mathbb{Q}(q)}$ :

$$
\begin{aligned}
& {\left[P_{i j}\right]=\left[P_{j i}\right], \quad i \cdot j=0,} \\
& {\left[P_{i j i}\right]=\left[P_{i^{(2)} j}\right]+\left[P_{j i^{(2)}}\right], \quad i \cdot j=-1,}
\end{aligned}
$$

that come from isomorphisms of left projective modules in Proposition 2.13. These equalities match the generators of the ideal $\mathcal{I}$. Therefore, the above homomorphism descends to a homomorphism

$$
\gamma_{\mathbb{Q}(q)}: \mathbf{f} \longrightarrow K_{0}(R)_{\mathbb{Q}(q)}
$$

Under this homomorphism induction of projective $R$-modules corresponds to the multiplication in ${ }_{\mathcal{A}} \mathbf{f}$, so that

$$
\gamma_{\mathbb{Q}(q)}\left(\theta_{i_{1}} \ldots \theta_{i_{k}}\right)=\left[P_{i_{1} \ldots i_{k}}\right]
$$

Passing to the divided powers shows that

$$
\gamma_{\mathbb{Q}(q)}\left(\theta_{i_{1}}^{\left(a_{1}\right)} \ldots \theta_{i_{k}}^{\left(a_{k}\right)}\right)=\left[P_{i_{1}^{\left(a_{1}\right)} \ldots i_{k}^{\left(a_{k}\right)}}\right]
$$

The bilinear forms on $\mathbf{f}$ and $K_{0}(R)_{\mathbb{Q}(q)}$ satisfy the same properties, listed earlier, and these properties uniquely determine the form on $\mathbf{f}$. Therefore, the homomorphism $\gamma_{\mathbb{Q}(q)}$ respects the bilinear forms:

$$
\left(\gamma_{\mathbb{Q}(q)}(x), \gamma_{\mathbb{Q}(q)}(y)\right)=(x, y)
$$

Since the bilinear form on $\mathbf{f}$ is nondegenerate, homomorphism $\gamma_{\mathbb{Q}(q)}$ is injective. The bar-involution on $\mathbf{f}$ is $q$-antilinear and fixes each product element $\theta_{i_{1}} \ldots \theta_{i_{k}}$. The bar-involution on $K_{0}(R)_{\mathbb{Q}(q)}$ is $q$-antilinear and fixes $\left[P_{i}\right]$ for each $\boldsymbol{i}$. Therefore, $\gamma_{\mathbb{Q}(q)}(\bar{x})=\overline{\gamma_{\mathbb{Q}(q)}(x)}$ for all $x \in \mathbf{f}$.

The image of the restriction of $\gamma_{\mathbb{Q}(q)}$ to ${ }_{\mathcal{A}} \mathbf{f}$ lies in $K_{0}(R)$, therefore we get a homomorphism $\gamma:{ }_{\mathcal{A}} \mathbf{f} \longrightarrow K_{0}(R)$ by restriction. This homomorphism is injective and satisfies all the properties stated in the proposition.

We will prove in the next section that $\gamma$ is an isomorphism. 
3.2. Surjectivity of $\gamma$. In this section we closely follow [25, Chapter 5]; all results there transfer directly to our case.

For $M$ in $R(\nu)-\bmod$ and $i \in I$ let

$$
\Delta_{i} M=\left(1_{\nu-i} \otimes 1_{i}\right) M={ }_{\nu-i, i} R_{\nu} \otimes_{R(\nu)} M,
$$

and, more generally,

$$
\Delta_{i^{n}} M=\left(1_{\nu-n i} \otimes 1_{n i}\right) M={ }_{\nu-n i, n i} R_{\nu} \otimes_{R(\nu)} M .
$$

We view $\Delta_{i^{n}}$ as the functor into the category $R(\nu-n i) \otimes R(n i)-\bmod$. There are functorial isomorphisms

(3.2) $\operatorname{HOM}_{R(\nu)}\left(\operatorname{Ind}_{\nu-n i, n i} N \otimes L\left(i^{n}\right), M\right) \cong \operatorname{HOM}_{R(\nu-n i) \otimes R(n i)}\left(N \otimes L\left(i^{n}\right), \Delta_{i^{n}} M\right)$, for $M$ as above and $N \in R(\nu-n i)-\bmod$. The following lemma is obvious.

\section{Lemma 3.5.}

$$
\operatorname{ch}\left(\Delta_{i^{n}} M\right)=\sum_{j \in \operatorname{Seq}(\nu-n i)} \operatorname{ch}\left(M, \ddot{j}^{n}\right) \cdot \boldsymbol{j},
$$

where we view $\Delta_{i^{n}} M$ as a module over the subalgebra $R(\nu-n i)$ of $R(\nu-n i) \otimes R(n i)$.

Let $\varepsilon_{i}(M)=\max \left\{n \geq 0 \mid \Delta_{i^{n}} M \neq 0\right\}$. This number is the length of the longest tail of $i$ 's in sequences $k$ with $1_{k} M \neq 0$.

Lemma 3.6. If $M \in R(\nu)-\bmod$ is irreducible, and $N \otimes L\left(i^{n}\right)\{r\}$ is an irreducible submodule of $\Delta_{i^{n}}(M)$ for some $0 \leq n \leq \varepsilon_{i}(M)$ and $r \in \mathbb{Z}$, then $\varepsilon_{i}(N)=\varepsilon_{i}(M)-n$.

Proof. This is our analogue of Lemma 5.1.2 of [25] and the proof is essentially the same. Let $\varepsilon=\varepsilon_{i}(M)$. Clearly, $\varepsilon_{i}(N) \leq \varepsilon_{i}(M)-n$. Isomorphisms (3.2) and the irreducibility of $M$ imply that it is a quotient of $\operatorname{Ind}_{\nu-n i, n i} N \otimes L\left(i^{n}\right)\{r\}$. By exactness of $\Delta_{i^{\varepsilon}} M$, we get that $\Delta_{i^{\varepsilon}}(M) \neq 0$ is a quotient of

$$
\Delta_{i \varepsilon}\left(\operatorname{Ind}_{\nu-n i, n i} N \otimes L\left(i^{n}\right)\right)\{r\} .
$$

Hence, the latter module is nonzero, and the inequality $\varepsilon_{i}(N) \geq \varepsilon_{i}(M)-n$ follows from the Shuffle lemma 2.20

Lemma 3.7. Suppose $N \in R(\nu)-\bmod$ is irreducible and $\varepsilon_{i}(N)=0$. Let $M=$ $\operatorname{Ind}_{\nu, n i} N \otimes L\left(i^{n}\right)$. Then

(1) $\Delta_{i^{n}} M \cong N \otimes L\left(i^{n}\right)$,

(2) hd $M$ is irreducible and $\varepsilon_{i}(\mathrm{hd} M)=n$,

(3) all other composition factors $L$ of $M$ have $\varepsilon_{i}(L)<n$.

Proof. This is the analogue of Lemma 5.1.3 in 25] for algebras $R(\nu)$.

(1) is immediate from the Shuffle lemma and Lemma 3.5.

(2) From (3.2) we see that a copy of $N \otimes L\left(i^{n}\right)$, possibly with a grading shift, appears in $\Delta_{i^{n}} Q$ for any nonzero quotient $Q$ of $M$, including direct summands of hd $M$. Part 1 , however, implies that $N \otimes L\left(i^{n}\right)$ appears only once in $\Delta_{i^{n}} M$, so that hd $M$ is irreducible.

(3) From part (2) we have $\Delta_{i^{n}}(M)=\Delta_{i^{n}}($ hd $M)$, so that $\Delta_{i^{n}}(L)=0$ for any other composition factor of $M$, since $\Delta_{i^{n}}$ is exact.

Lemma 3.8. Let $M \in R(\nu)-\bmod$ be irreducible and $\varepsilon=\varepsilon_{i}(M)$. Then $\Delta_{i \varepsilon} M$ is isomorphic to $N \otimes L\left(i^{\varepsilon}\right)$ for some irreducible $N \in R(\nu-\varepsilon i)-\bmod$ with $\varepsilon_{i}(N)=0$.

Proof. The proof is identical to that of Lemma 5.1.4 in 25]. 
Lemma 3.9. Let $N \in R(\nu)-\bmod$ be irreducible and $M=\operatorname{Ind}_{\nu, n i} N \otimes L\left(i^{n}\right)$. Then $\mathrm{hd} M$ is irreducible, $\varepsilon_{i}(\mathrm{hd} M)=\varepsilon_{i}(N)+n$, and all other composition factors $L$ of $M$ have $\varepsilon_{i}(L)<\varepsilon_{i}(N)+n$.

Proof. The same as the proof of Lemma 5.1.5 in [25].

Proposition 3.10. For any irreducible $M \in R(\nu)-\bmod$ and $0 \leq n \leq \varepsilon_{i}(M)$, $\operatorname{soc} \Delta_{i^{n}} M$ is an irreducible $R(\nu-n i) \otimes R(n i)$-module of the form $L \otimes L\left(i^{n}\right)$ with $\varepsilon_{i}(L)=\varepsilon_{i}(M)-n$.

Proof. The same as the proof of Theorem 5.1.6 in [25. The analogue of the Kato theorem in our framework is stated below (this theorem appears in the proof of Theorem 5.1.6).

Proposition 3.11. Let $\mu$ be a composition of $n$.

(1) The module $L\left(i^{n}\right)$ over the nil-Hecke algebra $R(n i)$ is the only graded irreducible module, up to isomorphism and graded shifts.

(2) All composition factors of $\operatorname{Res}_{\mu}^{n} L\left(i^{n}\right)$ are isomorphic to $L\left(i^{\mu_{1}}\right) \otimes \cdots \otimes L\left(i^{\mu_{r}}\right)$, up to grading shifts, and $\operatorname{soc}\left(\operatorname{Res}_{\mu}^{n} L\left(i^{n}\right)\right)$ is irreducible.

(3) $\operatorname{soc}\left(\operatorname{Res}_{n-1}^{n} L\left(i^{n}\right)\right) \cong L\left(i^{n-1}\right)$, up to a grading shift.

Here $\operatorname{Res}_{\mu}^{n}$ denotes the restriction to the parabolic nil-Hecke subalgebra $N H_{\mu} \cong$ $N H_{\mu_{1}} \otimes \cdots \otimes N H_{\mu_{r}}$. The proof in [25] works in this case as well, with the equivalent of Lemma 4.3.1 being Lemma 2.1.

Let $e_{i}=\operatorname{Res}_{\nu-i}^{\nu-i, i} \circ \Delta_{i}$ be the functor of composition of $\Delta_{i}$ with the restriction from $R(\nu-i) \otimes R(i)$ to $R(\nu-i)$. Then $\varepsilon_{i}(M)=\max \left\{n \geq 0 \mid e_{i}^{n} M \neq 0\right\}$ and

$$
\operatorname{Res}_{\nu-i}^{\nu} M=\bigoplus_{i \in I} e_{i} M
$$

Corollary 3.12. Let $M \in R(\nu)-\bmod$ be irreducible with $\varepsilon_{i}(M)>0$. Then $\operatorname{soc}\left(e_{i} M\right)$ is irreducible and $\varepsilon_{i}\left(\operatorname{soc}\left(e_{i} M\right)\right)=\varepsilon_{i}(M)-1$. Socles of $e_{i} M$ are pairwise nonisomorphic for different $i \in I$.

The proof is the same as for Corollaries 5.1.7 and 5.1.8 in [25].

For an irreducible $M \in R(\nu)-\bmod$ define

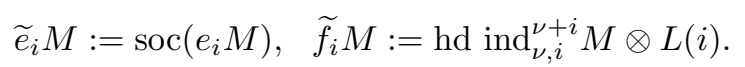

The module $\widetilde{f}_{i} M$ is irreducible by Lemma 3.9, while $\widetilde{e}_{i} M$ is irreducible or 0 by Corollary 3.12, and

$$
\varepsilon_{i}(M)=\max \left\{n \geq 0 \mid \widetilde{e}_{i}^{n} M \neq 0\right\}, \quad \varepsilon_{i}\left(\widetilde{f}_{i} M\right)=\varepsilon_{i}(M)+1 .
$$

In the statements below, isomorphisms of simple modules are allowed to be homogeneous (not necessarily degree-preserving).

Lemma 3.13. For an irreducible $M \in R(\nu)-\bmod$ we have

$$
\begin{aligned}
\operatorname{soc} \Delta_{i^{n}} M & \cong\left(\widetilde{e}_{i}^{n} M\right) \otimes L\left(i^{n}\right), \\
h d i n d_{\nu, n i}\left(M \otimes L\left(i^{n}\right)\right) & \cong \widetilde{f}_{i}^{n} M .
\end{aligned}
$$

Lemma 3.14. For an irreducible $M \in R(\nu)-\bmod$ the socle of $e_{i}^{n} M$ is isomorphic to $\widetilde{e}_{i}^{n} M^{\oplus[n] !}\left\{-\frac{n(n-1)}{2}\right\}$.

The proofs are equivalent to those of Lemmas 5.2.1 and 5.2.2 in [25]. 
Lemma 3.15. For irreducible modules $M \in R(\nu)-\bmod$ and $N \in R(\nu+i)-\bmod$ we have $\widetilde{f}_{i} M \cong N$ if and only if $\widetilde{e}_{i} N \cong M$.

The proof follows that of Lemma 5.2.3 in [25].

Corollary 3.16. Let $M, N \in R(\nu)-\bmod$ be irreducible. Then $\widetilde{f}_{i} M \cong \widetilde{f}_{i} N$ if and only if $M \cong N$. Assuming $\varepsilon_{i}(M), \varepsilon_{i}(N)>0, \widetilde{e}_{i} M \cong \widetilde{e}_{i} N$ if and only if $M \cong N$.

The character $\operatorname{ch}(M)$ of a finite-dimensional representation $M \in R(\nu)-\bmod$ takes values in $\mathbb{Z}\left[q, q^{-1}\right] \operatorname{Seq}(\nu)$, the free $\mathbb{Z}\left[q, q^{-1}\right]$-module generated by $\operatorname{Seq}(\nu)$, and descends to a homomorphism from $G_{0}(R(\nu))$ to $\mathbb{Z}\left[q, q^{-1}\right] \operatorname{Seq}(\nu)$.

Theorem 3.17. The character map

$$
\operatorname{ch}: G_{0}(R(\nu)) \longrightarrow \mathbb{Z}\left[q, q^{-1}\right] \operatorname{Seq}(\nu)
$$

is injective.

Equivalently, the characters of irreducible modules (one from each equivalence class up to grading shifts) are linearly independent functions on Seq $(\nu)$. The proof is identical to that of Theorem 5.3.1 in [25. Note that in our case the character of a finite-dimensional graded module is a function on sequences with values in $\mathbb{Z}\left[q, q^{-1}\right]$, while in the nongraded case of [25] its a function on sequences taking values in $\mathbb{Z}$. This discrepancy has no effect on the proof.

Passing to the fraction field $\mathbb{Q}(q)$ of $\mathbb{Z}\left[q, q^{-1}\right]$ and dualizing the map ch, which then becomes the composition

$$
\mathbb{Q}(q) \operatorname{Seq}(\nu) \longrightarrow \mathbf{f}_{\nu} \stackrel{\gamma_{\mathbb{Q}(q)}}{\longrightarrow} K_{0}(R(\nu))_{\mathbb{Q}(q)},
$$

we conclude that $\gamma_{\mathbb{Q}(q)}$, restricted to weight $\nu$, is a surjective map of $\mathbb{Q}(q)$-vector spaces. We have already observed that $\gamma$ and $\gamma_{\mathbb{Q}(q)}$ are injective. By summing over all weights, we obtain the following result.

Proposition 3.18. $\gamma_{\mathbb{Q}(q)}: \mathbf{f} \longrightarrow K_{0}(R)_{\mathbb{Q}(q)}$ is an isomorphism.

Therefore, the number of isomorphism classes of (graded) simple $R(\nu)$-modules is the same for any field $\mathbb{k}$.

Corollary 3.19. A (graded) irreducible $R(\nu)$-module is absolutely irreducible, for any $\Gamma, \mathbb{k}$ and weight $\nu$.

Next, assume that $\Gamma$ is finite. Choose a total order on $I, i(0)<i(1) \cdots<i(k-1)$, $k=|I|$. For $r>k$ define $i(r)=i\left(r^{\prime}\right)$ where $r^{\prime}$ is the residue of $r$ modulo $k$. Fix one representative $S_{b}$ from each isomorphism class $b$ of irreducible $R(\nu)$-modules, up to grading shifts. Recall that we denoted this set of isomorphism classes by $\mathbf{B}_{\nu}^{\prime}$. For all $\nu$, to each $b \in \mathbf{B}_{\nu}^{\prime}$ assign the following sequence $Y_{b}=y_{0} y_{1} \ldots$ of nonnegative integers: $y_{0}=\varepsilon_{i(0)}(M)$, and let $M_{1}=\widetilde{e}_{i(0)}^{y_{0}} M$. Inductively, $y_{r}=\varepsilon_{i(r)}\left(M_{r}\right)$, and $M_{r+1}=\widetilde{e}_{i(r)}^{y_{r}} M_{r}$. Note that $y_{0}+y_{1}+\cdots=|\nu|$ and only finitely many terms in the sequence are nonzero. Introduce a lexicographic order on sequences of nonnegative integers: $y_{0} y_{1} \cdots>z_{0} z_{1} \ldots$ if, for some $t, y_{0}=z_{0}, y_{1}=z_{1}, \ldots, y_{t-1}=z_{t-1}$ and $y_{t}>z_{t}$. This order induces a total order on $\mathbf{B}_{\nu}^{\prime}$, by $b>c$ iff $Y_{b}>Y_{c}$. To each sequence $Y_{b}=y_{0} y_{1} \ldots$ we assign the projective $R(\nu)$-module $P_{Y_{b}^{r}}$ associated to the divided powers sequence $Y_{b}^{r}=\cdots i(2)^{y(2)} i(1)^{y(1)} i(0)^{\left(y_{0}\right)}$ (the order of $y$ 's is reversed).

Proposition 3.20. $\operatorname{HOM}\left(P\left(Y_{b}\right), S_{c}\right)=0$ if $b<c$ and $\operatorname{HOM}\left(P\left(Y_{b}\right), S_{b}\right)=\mathbb{k}$. 
This follows from the previous results and implies that the image $[P]$ of any (graded) projective $R(\nu)$-module in the Grothendieck group $K_{0}(R(\nu))$ can be written as a linear combination, with coefficients in $\mathbb{Z}\left[q, q^{-1}\right]$, of images of divided powers projectives $\left[P_{\theta}\right]$, for divided power sequences $\theta$ of the form $Y_{b}^{r}$. Therefore, $\gamma:{ }_{\mathcal{A}} \mathbf{f} \longrightarrow K_{0}(R(\nu))$ is surjective. Since, $\gamma$ is also injective, it is an isomorphism. The case of an infinite $\Gamma$ follows by taking the direct limit of its finite subgraphs. This concludes the proof of the Theorem 1.1 stated in the introduction.

It would be interesting to find out if the theorem remains valid for rings $R(\nu)$ over $\mathbb{Z}$ rather than over a field $\mathbb{k}$.

For each divided power $i^{(a)}$ we have the corresponding projective $P_{i^{(a)}}$. Induction with this projective is an exact functor, denoted $\mathcal{F}_{i}^{(a)}$, from $R(\nu)-\bmod$ to $R(\nu+$ ai)-mod. Summing over all $\nu$, form the functor

$$
\mathcal{F}_{i}^{(a)}: R-\bmod \longrightarrow R-\bmod .
$$

This functor restricts to the subcategory $R$-pmod of the category of projective modules. To any divided power sequence $\theta=i_{1}^{\left(a_{1}\right)} \ldots i_{r}^{\left(a_{r}\right)}$ associate the functor

$$
\mathcal{F}_{\theta}=\mathcal{F}_{i_{1}}^{\left(a_{1}\right)} \circ \cdots \circ \mathcal{F}_{i_{r}}^{\left(a_{r}\right)}
$$

on $R$-mod. To a finite sum $\sum_{k} u_{k} \theta(k)$ where $u_{k} \in \mathbb{N}\left[q, q^{-1}\right]$ and $\theta(k)$ are divided powers sequences associate the direct sum of shifted copies of $\mathcal{F}_{\theta(k)}$ :

$$
\bigoplus_{k} \mathcal{F}_{\theta(k)}^{\oplus u_{k}}
$$

Theorem 3.21. For any relation

$$
\sum_{k} u_{k} \theta(k)=\sum_{\ell} v_{\ell} \theta^{\prime}(\ell)
$$

in ${ }_{\mathcal{A}} \mathbf{f}$ with positive coefficients $u_{k}, v_{\ell} \in \mathbb{N}\left[q, q^{-1}\right]$ there is an isomorphism of projectives

inducing an isomorphism of functors

$$
\bigoplus_{k} P_{\theta(k)}^{\oplus u_{k}} \cong \bigoplus_{\ell} P_{\theta^{\prime}(\ell)}^{\oplus v_{\ell}}
$$

$$
\bigoplus_{k} \mathcal{F}_{\theta(k)}^{\oplus u_{k}} \cong \bigoplus_{\ell} \mathcal{F}_{\theta^{\prime}(\ell)}^{\oplus v_{\ell}}
$$

This result follows immediately from the earlier ones.

We conclude that any relation in ${ }_{\mathcal{A}} \mathbf{f}$ lifts to an isomorphism of functors. It is natural to view the category $R$-pmod, as well as the category of induction functors on $R$-mod it gives rise to, as a categorification of ${ }_{\mathcal{A}} \mathbf{f}$, the integral form of the quantum universal enveloping algebra of the negative half of the simply-laced Kac-Moody algebra associated to the graph $\Gamma$.

The semilinear "hom" form $(,)^{\prime}$ on $K_{0}(R)$ defined by

$$
([P],[Q])^{\prime}:=\operatorname{gdimHOM}(P, Q)
$$

is related to the "tensor product" bilinear form $($, ) given by (2.45) via

$$
(x, y)^{\prime}=(\bar{x}, y) \text {. }
$$

Indeed, by surjectivity of $\gamma$, it suffices to check this relation for $x=\left[P_{i}\right]$ and $y=\left[P_{j}\right]$, in which case both sides are equal to the graded dimension of ${ }_{i} R(\nu)_{j}$. 
The involution $\sigma$ of $R(\nu)$ defined in Section 2.1 induces a self-equivalence of

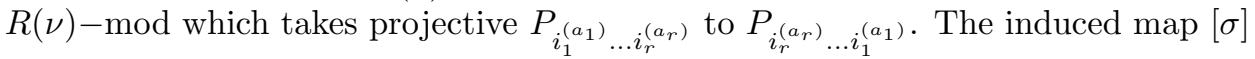
on the Grothendieck group $K_{0}(R)$ coincides, under the isomorphism $\gamma$, the $q$-linear anti-involution of ${ }_{\mathcal{A}} \mathbf{f}$ that fixes each $\theta_{i}^{(a)}$.

On the category $R(\nu)$-fmod we have the contravariant duality functor, which takes a finite-dimensional module $M$ to its vector space dual $M^{* \psi}$ twisted by the anti-involution $\psi$. This duality functor leaves invariant the character evaluated at $q=1$ :

$$
\operatorname{ch}\left(M^{* \psi}\right)_{q=1}=\operatorname{ch}(M)_{q=1} .
$$

Therefore, the contravariant duality preserves simples, up to overall shift:

$$
S_{b}^{* \psi} \cong S_{b}\{r\} .
$$

Given $\boldsymbol{i} \in \operatorname{Seq}(\nu)$, we have $\operatorname{ch}\left(S_{b}, \boldsymbol{i}\right) \in \mathbb{Z}\left[q^{2}, q^{-2}\right]$ or $\operatorname{ch}\left(S_{b}, \boldsymbol{i}\right) \in q \mathbb{Z}\left[q^{2}, q^{-2}\right]$ for parity reasons (more generally, this is true for any indecomposable object of $R(\nu)-\bmod$ and can be used to decompose $R(\nu)$-mod into the direct sum of two subcategories). Then $\operatorname{ch}\left(S_{b}^{* \psi}, \boldsymbol{i}\right) \in \mathbb{Z}\left[q^{2}, q^{-2}\right]$ if the same is true for $\operatorname{ch}\left(S_{b}, \boldsymbol{i}\right)$, and $\operatorname{ch}\left(S_{b}^{* \psi}, \boldsymbol{i}\right) \in$ $q \mathbb{Z}\left[q^{2}, q^{-2}\right]$ if $\operatorname{ch}\left(S_{b}, \boldsymbol{i}\right) \in q \mathbb{Z}\left[q^{2}, q^{-2}\right]$. Hence, the shift $r$ is an even number.

From now on we redefine $S_{b}$ by shifting its grading by $\frac{r}{2}$. We have $S_{b}^{* \psi} \cong S_{b}$ as graded modules. This normalization of $S_{b}$ does not depend on the choice of $i$. The character of $S_{b}$ is bar-invariant:

$$
\overline{\operatorname{ch}\left(S_{b}, \boldsymbol{i}\right)}=\operatorname{ch}\left(S_{b}, \boldsymbol{i}\right)
$$

for all $i \in \operatorname{Seq}(\nu)$, where $\bar{q}=q^{-1}$. Extending the bar-involution to $\mathbb{Z}\left[q, q^{-1}\right] \operatorname{Seq}(\nu)$ by $\overline{\boldsymbol{i}}=\boldsymbol{i}$, we have $\overline{\operatorname{ch}\left(S_{b}\right)}=\operatorname{ch}\left(S_{b}\right)$.

This canonical (balanced) choice of grading for $S_{b}$ allows us to fix the grading on indecomposable projective $P_{b}$ so that the quotient map $P_{b} \longrightarrow S_{b}$ is gradingpreserving. In this way we obtain a basis $\left\{\left[P_{b}\right]\right\}$ in ${ }_{\mathcal{A}} \mathbf{f}$ which depends only on the characteristic of $\mathbb{k}$. Both the multiplication and the comultiplication in this basis have coefficients in $\mathbb{N}\left[q, q^{-1}\right]$. An example below shows this basis to be different from the Lusztig-Kashiwara basis when $\Gamma$ is an odd length cycle and $\mathbb{k}$ has any characteristic, and when $\Gamma$ is a cycle and $\mathbb{k}$ has characteristic 2 .

3.3. Tight monomials and indecomposable projectives. Following Lusztig [35], we say that a monomial $\theta=\theta_{1}^{\left(a_{1}\right)} \ldots \theta_{k}^{\left(a_{k}\right)}$ is tight if it belongs to the canonical basis $\mathbf{B}$ of ${ }_{\mathcal{A}} \mathbf{f}$. It follows from the properties of the canonical basis that a monomial $\theta$ is tight if and only if $(\theta, \theta)-1 \in q \mathbb{N}[q]$ (or see [40, Proposition 3.1]).

Proposition 3.22. If a monomial $\theta$ is tight, the projective module $P_{\theta}$ is indecomposable.

Proof. Tightness of $\theta$ implies that $\operatorname{HOM}\left(P_{\theta}, P_{\theta}\right)$ is a $\mathbb{Z}_{+}$-graded $\mathbb{k}$-vector space which is one-dimensional in degree 0 . Therefore, any degree 0 endomorphism of $P_{\theta}$ is a multiple of the identity, and $P_{\theta}$ is indecomposable. This argument works even over $\mathbb{Z}$.

Example 3.23. When the graph $\Gamma$ consists of a single vertex $i$, the weight space ${ }_{\mathcal{A}} \mathbf{f}_{m i}$ is a rank one free $\mathbb{Z}\left[q, q^{-1}\right]$-module generated by $\theta_{i}^{(m)}$. The map $\gamma$ takes it to $\left[P_{i(m)}\right]$, the generator of the Grothendieck group $K_{0}(R(m i))$. Projective module $P_{i(m)}$ is indecomposable. 
Example 3.24. Let $\Gamma=\stackrel{i}{\circ} \stackrel{j}{\circ}$. Tight monomials $\theta_{i}^{(a)} \theta_{j}^{(b)} \theta_{i}^{(c)}(a, b, c \in \mathbb{N}, b \geq$ $a+c)$ and $\theta_{j}^{(c)} \theta_{i}^{(b)} \theta_{j}^{(a)}(a, b, c \in \mathbb{N}, b \geq a+c)$, with the identification

$$
\theta_{i}^{(a)} \theta_{j}^{(a+c)} \theta_{i}^{(c)}=\theta_{j}^{(c)} \theta_{i}^{(a+c)} \theta_{j}^{(a)}
$$

constitute the canonical basis $\mathbf{B}$ of ${ }_{\mathcal{A}} \mathbf{f}$; see [34, Example 14.5.4]. Therefore, images of indecomposable projectives $P_{i^{(a)} j^{(b)} i^{(c)}}, b \geq a+c$ and $P_{j^{(c)} i^{(b)} j^{(a)}}, b>a+c$, constitute a basis in the free $\mathbb{Z}\left[q, q^{-1}\right]$-module $K_{0}(R)$. Any indecomposable projective in $R$-mod is isomorphic to one of the above, up to a grading shift. Indecomposables $P_{i^{(a)} j^{(a+c)} i^{(c)}}$ and $P_{j^{(c)} i^{(a+c)} j^{(a)}}$ are isomorphic.

Example 3.25. Let $\Gamma=\overbrace{0}$ be a cycle with $n \geq 3$ vertices. Label the vertices clockwise by $1,2, \ldots, n$ and let $\boldsymbol{i}=12 \ldots n$. Then $\operatorname{HOM}\left(P_{\boldsymbol{i} i}, P_{\boldsymbol{i} i}\right)$ is $\mathbb{Z}_{+}$-graded and $\operatorname{Hom}\left(P_{i \boldsymbol{i}}, P_{\boldsymbol{i} i}\right)$ is 2 -dimensional with the basis $\left\{{ }_{i i} 1_{i \boldsymbol{i}}, \alpha\right\}$, where

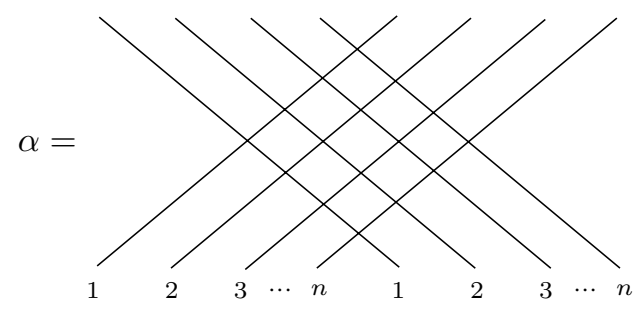

A computation shows that $\operatorname{deg}(\alpha)=0$ and

$$
\alpha^{2}=\left\{\begin{array}{cl}
0 & \text { if } n \text { is odd } \\
-2 \alpha & \text { if } n \text { is even; }
\end{array}\right.
$$

implying that ${ }_{i i} 1_{i i}$ is the only idempotent in $P_{i i}$ if $n$ is odd, or if $n$ is even and $\operatorname{char}(\mathbb{k})=2$. Under these assumptions, $P_{\boldsymbol{i} i}$ is indecomposable, but

$$
\left(\left[P_{i i}\right],\left[P_{i i}\right]\right) \in 2+q \mathbb{N}[q] \neq 1+q \mathbb{N}[q]
$$

and $\left[P_{i i}\right]$ is not a canonical basis element. For $\Gamma$, an odd length cycle, we found an indecomposable projective $P_{\boldsymbol{i} i}$ whose image in the Grothendieck group is not a canonical basis vector, while being invariant under the bar involution: $\overline{\left[P_{i i}\right]}=\left[P_{i i}\right]$.

When $n$ is even and $\operatorname{char}(\mathbb{k}) \neq 2, \alpha_{0}=-\frac{\alpha}{2}$ is an idempotent in $\operatorname{Hom}\left(P_{\boldsymbol{i} i}, P_{\boldsymbol{i} i}\right)$, and $P_{i i} \cong P_{i i} \alpha_{0} \oplus P_{i i}\left(1-\alpha_{0}\right)$ is isomorphic to the direct sum of two indecomposable projectives. Furthermore, $\alpha=\beta_{1} \beta_{0}$ where

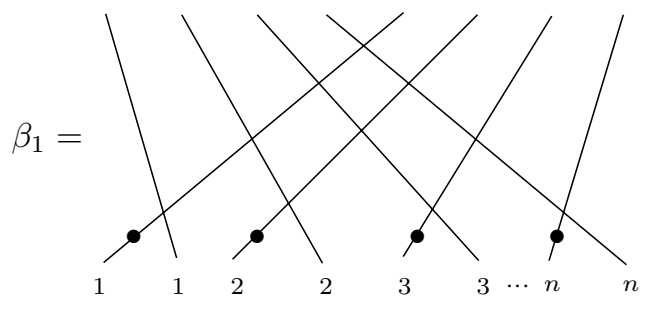




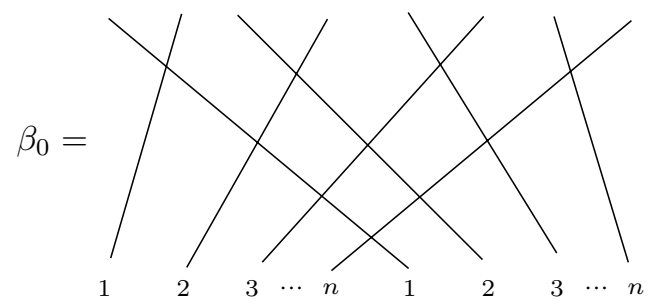

Module homomorphisms

$$
\begin{aligned}
& \beta_{1}: \quad P_{i i} \longrightarrow P_{1^{(2)} 2^{(2)} \ldots n^{(2)}}, \\
& \beta_{0}: \quad P_{1^{(2)} 2^{(2)} \ldots n^{(2)} \longrightarrow P_{i i},},
\end{aligned}
$$

induced by these elements via right multiplication have degree 0 , and $\beta_{0} \beta_{1}=-2 \cdot \mathrm{Id}$, so that $P_{i i} \alpha_{0} \cong P_{1^{(2)} 2^{(2)} \ldots n^{(2)} \text {. }}$.

3.4. A conjecture on categorification of irreducible representations. Choose $\lambda \in \mathbb{N}[I], \lambda=\sum \lambda_{i} \cdot i, i \in I$. Let $R(\nu ; \lambda)$ be the quotient ring of $R(\nu)$ by the ideal generated by all diagrams of the form

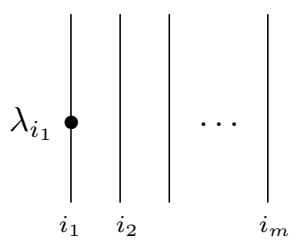

where $i_{1} \ldots i_{m} \in \operatorname{Seq}(\nu)$ and the leftmost string has $\lambda_{i_{1}}$ dots on it. The ring $R(\nu ; \lambda)$ inherits a grading from $R(\nu)$. These quotient rings should be the analogues of the Ariki-Koike cyclotomic Hecke algebras in our framework. Let

$$
R(* ; \lambda) \stackrel{\text { def }}{=} \bigoplus_{\nu \in \mathbb{N}[I]} R(\nu ; \lambda),
$$

and switch from $\mathbb{Z}$ to a field $\mathbb{k}$. We expect that, for sufficiently nice $\Gamma$ and $\mathbb{k}$, the category of graded modules over $R(* ; \lambda)$ categorifies the integrable irreducible $U_{q}(\mathfrak{g})$-representation $V_{\lambda}$ with the highest weight $\lambda$. Let $R(\nu ; \lambda)-$ pmod be the category of finitely-generated graded projective left $R(\nu ; \lambda)$-modules and

$$
R(* ; \lambda)-\operatorname{pmod} \stackrel{\text { def }}{=} \bigoplus_{\nu \in \mathbb{N}[I]} R(\nu ; \lambda)-\text { pmod. }
$$

There should exist an isomorphism

$$
K_{0}(R(* ; \lambda)) \cong V_{\mathbb{Z}, \lambda},
$$

where $V_{\mathbb{Z}, \lambda}$ is an integral version of $V_{\lambda}$, a free $\mathbb{Z}\left[q, q^{-1}\right]$-module spanned by the compositions of divided differences $F_{i}^{(a)}$ applied to the highest weight vector $v_{\lambda} \in V_{\lambda}$. Under this isomorphism indecomposable projectives should correspond to canonical basis vectors in $V_{\lambda}$. The action of $E_{i}^{(a)}$ and $F_{i}^{(a)}$ should lift to exact functors $\mathcal{E}_{i}^{(a)}$ and $\mathcal{F}_{i}^{(a)}$ between categories $R(\nu ; \lambda)-\operatorname{pmod}$ and $R(\nu+a i ; \lambda)-$ pmod as well as the categories $R(\nu ; \lambda)-\bmod$ and $R(\nu+a i ; \lambda)-\bmod$ of all finitely-generated graded modules. These functors $\mathcal{E}_{i}^{(a)}$ and $\mathcal{F}_{i}^{(a)}$ will be direct summands of the induction 
and restriction functors between $R(\nu ; \lambda)$ and $R(\nu+a i ; \lambda)$-modules, defined á la Ariki. We expect them to be biadjoint, up to grading shifts.

\section{ACKNOWLEDGMENTS}

We thank J. Brundan and A. Kleshchev for pointing out reference 38 to us. This paper was written while the first author was at the Institute for Advanced Study, and he would like to thank the Institute for its hospitality and the NSF for fully supporting him during that time through the grant DMS-0635607. Additional partial support came from the NSF grant DMS-0706924.

\section{REFERENCES}

[1] S. Ariki. On the decomposition numbers of the Hecke algebra of $G(m, 1, n)$. J. Math. Kyoto Univ., 36(4):789-808, 1996. MR 1443748 (98h:20012)

[2] S. Ariki. Lectures on cyclotomic Hecke algebras,Quantum groups and Lie theory, London Math. Soc. Lecture Notes Ser., 290, Cambridge Univ. Press, Cambridge, 2001. MR1903956 (2004a:20009)

[3] S. Ariki. Representations of quantum algebras and combinatorics of Young tableaux, volume 26 of University Lecture Series. AMS, Providence, RI, 2002.

[4] A. Beilinson, G. Lusztig, and R. MacPherson. A geometric setting for the quantum deformation of $\mathrm{GL}_{n}$. Duke Math. J., 61(2):655-677, 1990. MR:1074310 (91m:17012)

[5] I. Bernstein, I. Gelfand, and S. Gelfand. Schubert cells, and the cohomology of the spaces G/P. Russian Math. Surveys, 28:1-26, 1973. MR0429933(55:2941)

[6] J. Bernstein, I. B. Frenkel, and M. Khovanov. A categorification of the Temperley-Lieb algebra and Schur quotients of $\mathrm{U}(\mathrm{sl}(2))$ via projective and Zuckerman functors. Selecta Math. (N.S.), 5(2):199-241, 1999. MR.1714141 (2000i:17009)

[7] S. Billey and V. Lakshmibai. Singular loci of Schubert varieties, volume 182 of Progress in Mathematics. Birkhäuser Boston, Inc., Boston, MA, 2000. MR.1782635(2001j:14065)

[8] J. Brundan and A. Kleshchev. Hecke-Clifford superalgebras, crystals of type $A_{2 l}^{(2)}$ and modular branching rules for $\widehat{S}_{n}$. Represent. Theory, 5:317-403 (electronic), 2001, MR 1870595 (2002j:17024)

[9] J. Chuang and R. Rouquier. Derived equivalences for symmetric groups and $\mathfrak{s l}_{2}-$ categorification. Ann. of Math., 167:245-298, 2008. MR.2373155(2008m:20011)

[10] L. Crane and I. B. Frenkel. Four-dimensional topological quantum field theory, Hopf categories, and the canonical bases. J. Math. Phys., 35(10):5136-5154, 1994. MR.1295461 (96d:57019)

[11] M. Demazure. Invariants symétriques entiers des groupes de Weyl et torsion. Invent. Math., 21:287-301, 1973. MR0342522 (49:7268)

[12] V. Drinfeld. Quantum groups. In Proceedings of the International Congress of Mathematicians, Vol. 1, 2 (Berkeley, Calif., 1986), pages 798-820, Providence, RI, 1987. Amer. Math. Soc. MR934283 (89f:17017)

[13] I. B. Frenkel, M. Khovanov, and C. Stroppel. A categorification of finite-dimensional irreducible representations of quantum $\mathrm{sl}(2)$ and their tensor products. Selecta Math. (N.S.), 12(3-4):379-431, 2006. MR2305608 (2008a:17014)

[14] I. Grojnowski. Affine $s l_{p}$ controls the representation theory of the symmetric group and related Hecke algebras, 1999, math.RT/9907129.

[15] I. Grojnowski and G. Lusztig. On bases of irreducible representations of quantum $\mathrm{GL}_{n}$. In Kazhdan-Lusztig theory and related topics (Chicago, IL, 1989), volume 139 of Contemp. Math., pages 167-174. 1992. MR1197834 (94a:20070)

[16] I. Grojnowski and G. Lusztig. A comparison of bases of quantized enveloping algebras. In Linear algebraic groups and their representations (Los Angeles, CA, 1992), volume 153 of Contemp. Math., pages 11-19. 1993. MR1247495(94m:17012)

[17] I. Grojnowski and M. Vazirani. Strong multiplicity one theorems for affine Hecke algebras of type A. Transform. Groups, 6(2):143-155, 2001. MR1835669(2002c:20008)

[18] M. Jimbo. A $q$-difference analogue of $U(g)$ and the Yang-Baxter equation. Lett. Math. Phys., 10(1):63-69, 1985. MR797001 (86k:17008) 
[19] M. Kashiwara. Crystalizing the $q$-analogue of universal enveloping algebras. Comm. Math. Phys., 133(2):249-260, 1990. MR.1090425 (92b:17018)

[20] M. Kashiwara. On crystal bases of the $Q$-analogue of universal enveloping algebras. Duke Math. J., 63(2):465-516, 1991. MR1115118 (93b:17045)

[21] M. Khovanov. Nilcoxeter algebras categorify the Weyl algebra. Comm. Algebra, 29(11):50335052, 2001. MR1856929 (2002h:16041)

[22] A. Kleshchev. Branching rules for modular representations of symmetric groups. II. J. Reine Angew. Math., 459:163-212, 1995. MR1319521 (96m:20019b)

[23] A. Kleshchev. Branching rules for modular representations of symmetric groups. III. Some corollaries and a problem of Mullineux. J. London Math. Soc. (2), 54(1):25-38, 1996. MR1395065 (96m:20019c)

[24] A. Kleshchev. On decomposition numbers and branching coefficients for symmetric and special linear groups. Proc. London Math. Soc. (3), 75(3):497-558, 1997. MR1466660 (98g:20026)

[25] A. Kleshchev. Linear and projective representations of symmetric groups, volume 163 of Cambridge Tracts in Mathematics. Cambridge U. Press, 2005. MR2165457 (2007b:20022)

[26] B. Kostant and S. Kumar. The nil Hecke ring and cohomology of $G / P$ for a Kac-Moody group G. Adv. in Math., 62(3):187-237, 1986. MR866159(88b:17025b)

[27] A. Lascoux, B. Leclerc, and J.-Y. Thibon. Hecke algebras at roots of unity and crystal bases of quantum affine algebras. Comm. Math. Phys., 181(1):205-263, 1996. MR.1410572 (97k:17019)

[28] A. D. Lauda. A categorification of quantum sl(2), 2008, arXiv:0803.3652.

[29] A. D. Lauda. Categorified quantum sl(2) and equivariant cohomology of iterated flag variaties, 2008, arXiv:0803.3848.

[30] B. Leclerc. Dual canonical bases, quantum shuffles and q-characters. Math. Z. 246(4):691-732 (2004), no. 4. MR2045836 (2005c:17019)

[31] G. Lusztig. Canonical bases arising from quantized enveloping algebras. J. Amer. Math. Soc., 3(2):447-498, 1990. MR.1035415 (90m:17023)

[32] G. Lusztig. Canonical bases arising from quantized enveloping algebras. II. Progr. Theoret. Phys. Suppl., 102:175-201 (1991), 1990. Common trends in mathematics and quantum field theories (Kyoto, 1990). MR1182165 (93g:17019)

[33] G. Lusztig. Quivers, perverse sheaves, and quantized enveloping algebras. J. Amer. Math. Soc., 4(2):365-421, 1991. MR 1088333 (91m:17018)

[34] G. Lusztig. Introduction to quantum groups, volume 110 of Progress in Mathematics. Birkhäuser Boston, Inc., Boston, MA, 1993. MR1227098 (94m:17016)

[35] G. Lusztig. Tight monomials in quantized enveloping algebras. In Quantum deformations of algebras and their representations (Ramat-Gan, 1991/1992; Rehovot, 1991/1992), volume 7 of Israel Math. Conf. Proc., pages 117-132. Bar-Ilan Univ., 1993. MR.1261904 (95i:17016)

[36] L. Manivel. Symmetric functions, Schubert polynomials and degeneracy loci, volume 6 of SMF/AMS Texts and Monographs. AMS, Providence, RI, 2001.

[37] A. Mathas. Iwahori-Hecke algebras and Schur algebras of the symmetric group, volume 15 of University Lecture Series. AMS, Providence, RI, 1999. MR.1711316 (2001g:20006)

[38] C. Năstăsescu and F. Van Oystaeyen. Methods of graded rings, volume 1836 of Lecture Notes in Mathematics. Springer-Verlag, Berlin, 2004. MR2046303 (2005d:16075)

[39] A. Postnikov. Enumeration in algebra and geometry. Ph.D. thesis, MIT, 1997 available at http://citeseer.ist.psu.edu/postnikov97enumeration.html.

[40] M. Reineke. Monomials in canonical bases of quantum groups and quadratic forms. J. Pure Appl. Algebra, 157(2-3):301-309, 2001. MR1812057(2002e:17022)

[41] C. Ringel. Hall algebras and quantum groups. Invent. Math., 101(3):583-591, 1990. MR:1062796 (91i:16024)

[42] R. Rouquier. Higher representation theory. Talk at IAS, March 2008.

[43] R. Rouquier. Higher representations of Kac-Moody algebras. Work in progress.

[44] J. Sussan. Category O and sl(k) link invariants, 2007, math.QA/0701045.

[45] M. Vazirani. Irreducible modules over the affine Hecke algebra: a strong multiplicity one result. Ph.D. thesis, UC Berkeley, 1999, math.RT/0107052.

[46] M. Vazirani. Parameterizing Hecke algebra modules: Bernstein-Zelevinsky multisegments, Kleshchev multipartitions, and crystal graphs. Transform. Groups, 7(3):267-303, 2002. MR:1923974 (2003g:20009) 
[47] A. Zelevinsky. Induced representations of reductive p-adic groups. II. On irreducible representations of GL(n). Ann. Sci. École Norm. Sup. (4), 13(2):165-210, 1980. MR584084 (83g:22012)

[48] H. Zheng. A geometric categorification of tensor products of $U_{q}\left(s l_{2}\right)$-modules, 2007, arXiv:0705.2630.

[49] H. Zheng. Categorification of integrable representations of quantum groups, 2008, arXiv:0803.3668.

School of Mathematics, Institute for Advanced Study, Princeton, New Jersey 08540

Current address: Department of Mathematics, Columbia University, New York, New York 10027

E-mail address: khovanov@math.columbia.edu

Department of Mathematics, Columbia University, New York, New York 10027

E-mail address: lauda@math.columbia.edu 